\title{
Population Differentiation of Rhodobacteraceae Along Coral Compartments
}

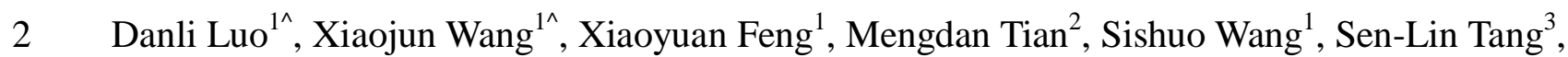
Put Ang $\mathrm{Jr}^{4}$, Aixin $\mathrm{Yan}^{2}$, Haiwei Luo ${ }^{1 *}$

4

7

8

8 SAR

$9 \quad{ }^{3}$ Biodiversity Research Center, Academia Sinica, Taipei, 11529, Taiwan

$10{ }^{4}$ Institute of Space and Earth Information Science, The Chinese University of Hong Kong, Hong

11 Kong SAR

12

13 'These authors contribute equally to this work.

14

\section{*Corresponding author:}

Haiwei Luo

The Chinese University of Hong Kong

Shatin, Hong Kong SAR

Phone: (+852) 39436121

E-mail: hluo2006@gmail.com 


\section{Abstract}

Coral mucus, tissue and skeleton harbor compositionally different microbiota, but how

27 these coral compartments shape the microbial evolution remains unexplored. Here, we focused on the Rhodobacteraceae, which represents a significant but variable proportion (5-50\%) of the coral microbiota. We sequenced 234 genomes constituting two divergent populations inhabiting a prevalent coral species Platygyra acuta. One population diverged into two clades colonizing the

31 mucus and skeleton respectively. We reconstructed the ancestral gene changing events that

32 potentially drove the split, and found that the affected genes matched well with the distinct

33 physicochemical features of the mucus and skeleton. Specifically, the mucus clade acquired

34 functions involved in the utilization of coral osmolytes abundant in the mucus (e.g.,

35 methylamines, DMSP, taurine and L-proline), whereas the skeleton clade uniquely harbored

36 traits that may promote adaptation to the low-energy and diurnally anoxic skeleton (e.g., sulfur

37 oxidation and swimming motility). These between-clade genetic differences were largely

38 supported by physiological assays. Expanded analyses by including relatives isolated from

39 various marine environments suggest that the mucus and skeleton clades may have diversified in

40 non-coral habitats, but they also consolidated a key role of distinct coral compartments in

41 diversifying many of the above-mentioned traits. The second population varied only at a few

42 dozen nucleotide sites across the whole genomes, and the Slatkin-Maddison test supported that

43 dispersal limitation between coral compartments is another key mechanism driving microbial

44 population differentiation. Collectively, our results suggest that different coral compartments

45 represent ecologically distinct and microgeographically separate habitats that drive the evolution 46 of the coral microbiota. 


\section{Introduction:}

As the home to more than $25 \%$ of marine life, coral reefs are an important habitat promoting biodiversity in the ocean [1]. The coral holobiont is a dynamic community of coral

51 polyps, microalgae, fungi, prokaryotes and viruses [2]. Scleractinian corals harbor three major

52 compartments microbial populations inhabit (Fig. S1), namely coral mucus, skeleton and tissue

53 [2]. The coral mucus is a nutrient and energy-rich mixture of materials [3, 4], and its primary

54 structural macromolecules are glycoproteins, polysaccharides and lipids [5, 6]. In addition,

55 mucus contains rich organic osmolytes secreted by the coral host and its endosymbiont

56 Symbiodiniaceae [7]. The osmolytes are compatible or counteracting solutes that can be

57 accumulated in the cells in response to the fluctuation of osmotic stresses, thermal stress and

58 hypoxia [8, 9], which includes methylamines [e.g., glycine betaine (GBT), choline,

59 choline-O-sulfate (COS), sarcosine and trimethylamine N-oxide (TMAO)], methyl sulfonium

60 [e.g., dimethylsulfoniopropionate (DMSP) and dimethyl sulfoxide (DMSO)], amino acid

61 derivatives (e.g., taurine, L-proline and ectoine), sugars (e.g., trehalose and arabinose) and

62 polyols (e.g., glycerol) [9]. Of these, GBT, taurine and DMSP represent the major osmolytes of

63 corals [10]. The coral animals eliminate or accumulate the osmolytes quickly to equilibrate the

64 fluctuated salinity caused by, for example, tides, air exposure, evaporation, and heavy rainfalls

65 [11]. These processes together lead to the enrichment of wasted osmolytes in mucus [12], which

66 serve as organic nutrients to support the resident bacteria [13, 14]. In contrast, the skeleton is a

67 low-energy environment embedded in the coral tissue, where up to $99 \%$ of the photosynthetically

68 active radiation is either scattered or entrapped by the Symbiodiniaceae located at coral tissue

69 [15]. The porous structure of the skeleton is mainly constructed with inorganic aragonite crystals

70 along with a small fraction $(\leq 0.1 \%$ by weight $)$ of the organic matrix $[16,17]$ which limits 
71 skeleton-associated microbes [18]. Although the light penetration is low, there are still endolithic

72 algae that reside inside the skeleton, whose photosynthesis and respiration generate a diel

73 fluctuation of the oxygen and $\mathrm{pH}$ levels in the skeleton [15]. Likewise, the Symbiodiniaceae in

74 the coral cells causes similar diel changes of the tissue [19].

The fact that different coral compartments are featured with distinct physicochemical

76 conditions indicates that coral is an ideal system for studying microbial niche adaptation.

77 Previous studies suggest that the community structure of the bacterial associates are largely

78 shaped by the coral anatomy $[20,21]$. Among the three compartments, for example, the mucus

79 harbors a higher proportion of compartment-specific bacteria than the tissue [22] and the

80 skeleton accommodates a microbial community of greater diversity compared to the tissue and

81 mucus $[15,23]$. While these studies illustrated potentially important differences in terms of the

82 microbial identities and functions across the coral compartments, they provided limited

83 taxonomic resolution in functional differentiation along coral compartments. Since functional

84 differences can be ascribed to both environmental heterogeneity and phylogenetic diversity, a

85 robust association of metabolic functions with ecological niches may be established when the

86 phylogenetic distance of lineages under comparison is minimized. Leveraging the single

87 nucleotide polymorphism (SNP) distribution at the core genome and gene frequency at the

88 accessory genome, the culture-based population genomics is able to uncover the mechanisms

89 driving population differentiation and niche adaptation [24-27]. To our knowledge, this approach

90 has not been used to investigate coral-associated microbial populations.

91 Among the numerous bacterial groups found on corals, Ruegeria is among the top three

92 genera that are most widely associated with coral species [28]. Ruegeria is a genus affiliated with

93 alphaproteobacterial family Rhodobacteraceae. Members of Ruegeria likely establish 
94 mutualistic interactions with the coral hosts. For instance, they are constantly associated with

95 both broadcast-spawning and brooding corals in their early developmental stages [29], and they

96 are one of the key bacterial taxa that can be vertically transferred from parents to larvae in the

97 brooding coral Pocillopora damicornis [30]. Some Ruegeria strains also help the larval

98 settlement in octocoral species [31]. In adult corals, Ruegeria strains can inhibit the growth of

99 pathogenic Vibrio species [32]. Nevertheless, some Ruegeria members may be potential

100 opportunistic pathogens, which show increased abundance when coral hosts are under stress

$101[33-35]$.

102 Platygyra acuta, the scleractinian coral sampled in the present study, is known for its

103 high tolerance to temperature and salinity stresses, as well as its resistance to bleaching in winter

104 [36]. It is not only the dominant species in Hong Kong coastal waters, but also a globally

105 important germplasm resource to understand the survival of marginal reefs $[37,38]$. We

106 harvested two Rhodobacteraceae populations from P. acuta: one was a member of Ruegeria

107 (hereafter, the Ruegeria population) and the other cannot be assigned to any described genus in

108 the Rhodobacteraceae (hereafter, the Rhodobacteraceae population). Both populations are

109 members of the Roseobacter group, a phylogenetic group encompassing the most taxonomic

110 diversity found in the Rhodobacteraceae [39]. The two populations consisted of 20 and 214

111 isolates, respectively. Members of the Ruegeria population harbored a great amount of genetic

112 variation, and their phylogenetic clustering and metabolic differences matched well with the

113 coral compartment differentiation. While members of the Rhodobacteraceae population varied at

114 only a few dozen SNP sites across the whole genomes, they nevertheless displayed a

115 microbiogeographic pattern across coral compartments, which is a signature of population

116 differentiation due to dispersal limitation. 


\section{Materials and Methods:}

Samples of $P$. acuta and ambient seawater were collected by SCUBA diving in Hong

120 Kong waters from April 2017 to February 2018. Bacteria were isolated from the three coral

121 compartments including mucus, skeleton and tissue, and also from the ambient seawater. We

122 identified two populations representing divergent lineages of Rhodobacteraceae suitable for

123 subsequent population genomic analyses according to two criteria: i) there were a large number

124 of isolates within each population and ii) multiple coral compartments were sampled in each

125 population. One population is a member of the ubiquitous coral-associated genus Ruegeria (20

126 isolates), and the other cannot be assigned to any described genus and was thus termed the

127 Rhodobacteraceae population (214 isolates). Genomes of all these 234 isolates were sequenced

128 with BGISEQ-500 PE100, assembled and annotated. The strain HKCCD4315 in the Ruegeria

129 population was additionally sequenced using the PacBio Sequel platform to obtain a closed

130 genome.

131 The Ruegeria population was initially analyzed to understand its phylogenetic and

132 population genetic structure. The orthologous gene families were identified with OrthoFinder

133 v2.2.1 [40] and the maximum likelihood phylogenomic tree was constructed based on the

134 concatenated single-copy gene alignments at the amino acid level using IQ-TREE v1.6.5 [41].

135 Two clades (namely, clade-M and clade-S) matched well with the coral mucus and skeleton

136 compartments, respectively, along with an additional clade locating at the outgroup position. The

137 pairwise $16 \mathrm{~S}$ rRNA gene identity and the whole-genome average nucleotide identity (ANI)

138 between- and within-clades were compared to help determine whether speciation has already

139 occurred between these clades [42]. Phylogenetic separation does not necessarily lead to allelic 
140 isolation between the phylogenetic clusters $[43,44]$. We therefore investigated the population

141 structure of clade-M and clade-S with the coancestry analysis implemented in fineSTRUCTURE

142 v2.0.7 [45], and characterized the potential gene flow barrier by comparing the between-clade to

143 within-clade relative frequency of recombination to point mutation $(\rho / \theta)$ [46] and relative effect

144 of recombination to mutation $(\mathrm{r} / \mathrm{m})$ [46], and by determining the fixation index $\left(\mathrm{F}_{\mathrm{st}}\right)$ across the

145 core genome shared by clade-M and clade-S [47]. We further tested whether the genetic

146 separation of the two clades occurred at the accessory genome by measuring the similarity of

147 genome content among strains with the Jaccard index and subsequently clustering it with the

148 complete-linkage method [27].

150 was further investigated at the functional level. We sought to identify signature genes of potential

151 importance in niche adaptation from both core and accessory genome. In the core genome, novel

152 allelic replacements via homologous recombination with external species at certain core loci

153 represent a potentially important and prevalent adaptive mechanism in marine bacterial

154 populations $[44,48]$. This type of core genes was also identified here. Briefly, the substitution

155 rate at synonymous (silent and thus largely neutral) sites $\left(d_{S}\right)$ across all single-copy core genes

156 and across all pairwise comparisons between isolates from clade-M and clade-S was clustered

157 using the K-means method, which resulted in a cluster composed of genes each showing

158 unusually large $d_{S}$ values, the genetic signature of an evolution history subjected to novel allelic

159 replacement [48]. For the identified core genes, gene trees were built and compared to the genome

160 tree to help determine which clade was subjected to novel allelic replacement. In terms of the

161 accessory genome, we focused on the genes largely specific to clade-M (or clade-S) and tried to

162 resolve two competing hypotheses: whether they resulted from acquisition events at the last 
163 common ancestor (LCA) of clade-M (or clade-S) or loss events at the LCA of clade-S (or

164 clade-M). Specifically, the gene gain and loss history of clade-specific genes were inferred based

165 on the gene copy number distribution among extant genomes of the Ruegeria population using

166 BadiRate v1.35 [49]. and checked manually based on the distribution pattern of pseudogenes in

167 the context of gene clusters [50]. For genes whose functions were potentially relevant to niche

168 adaptation and which differentiated the clade-M and clade-S at the DNA level, their potential

169 functional outcomes were tested using physiological assays, though we did not have direct

170 evidence connecting the genetic and phenotypic differentiation.

171 To test whether the clade-M and clade-S may have evolved from a single ancestor

172 initially colonized on the coral or these two clades may have already split in marine habitats

173 unrelated to corals and subsequently independently colonized coral compartments, we sampled

174 and sequenced 22 closely related Ruegeria isolates mostly from other marine habitats in Hong

175 Kong, constructed an expanded phylogenomic tree, and inferred the evolutionary history of the

176 ecological relevant genes using the above-mentioned methods. For the flagellar gene cluster, its

177 evolutionary history cannot be simply resolved based on the gene copy number distribution

178 pattern. In this case, pseudogenes may provide unequivocal evidence for the presence of this

179 gene cluster in the ancestral populations, and they were identified following a previous study

180 [50]. We additionally estimated the potential time of the split between clade-M and clade-S based

181 on synonymous substitution rate, spontaneous mutation rate and potential generation time in the

182 wild, and the goal of this analysis to show its antiquity compared to the coral ages.

183 For the Rhodobacteraceae population, the genetic uniformity enabled the

184 Slatkin-Maddison test [51] to measure the degree of compartmentalization on population 
185 subdivision. We also estimated the time of its origin and compared it to the potential age of the

186 coral animal. All the methodological details were described in Supplementary Text 1.

188 Results and Discussion:

189 Features of a Ruegeria population isolated from different coral compartments

190 The phylogenomic tree showed that the 20 closely related Ruegeria strains isolated from

$191 P$. acuta formed three clades (Fig. 1A). Among these, the six clade-S members were all isolated

192 from the skeleton, and the six clade-M members were all collected from mucus except for one

193 from ambient seawater (Fig. 1A). The outgroup clade consisted of seven skeleton-associated

194 strains and one tissue-associated strain. As these strains were isolated from the same coral

195 species, interpretation of any strain-level genomic differences ruled out the host species effect.

196 Therefore, our finding of the phylogenetic clustering by their source compartments indicates that

197 adaptation to the distinct coral compartments likely drives the evolutionary diversification of this

198 Ruegeria population [23].

199 We further investigated the ANI and 16S rRNA gene identity for these 20 strains, which

200 are standard measures typically used for bacteria species delineation [42, 52]. The genetic

201 clusters based on ANI or 16S rRNA genes are consistent with the phylogenetic clustering (Fig.

202 1B). Within each clade, both ANI and 16S rRNA gene identities are greater than the level often

203 used to delineate a bacterial species at 95.0\% [52] and 98.7\% [42], respectively. Between

204 clade-M and clade-S, ANI is lower than 89.9\% (Table 1, Fig. 1B), but the average 16S rRNA

205 gene identity is greater than $99.7 \%$ (Table 1, Fig. 1B). Considering that the 16S rRNA genes may

206 not provide sufficient resolution at the species level [53], we hypothesized that each clade may 
207 represent a distinct species and that the speciation process may have already completed. We

208 tested this hypothesis as follows.

210 Differentiation of the Ruegeria population along coral compartments at the core genome

211 The branching pattern of a phylogenetic tree cannot always represent the population

212 structure as the rate and influence of recombination may significantly affect the evolution of

213 bacterial species [43]. To elucidate whether the population subdivision occurred between

214 clade-M and clade-S, fineSTRUCTURE v2.0.7 [45] was applied to the core genome alignment

215 of the two clades. As a result, 12 strains from clade-S and clade-M were assigned to seven

216 subpopulations, three in clade-S and four in clade-M (Fig. 1C). The proportion of co-ancestry

217 shared within each clade is much greater than that between clades (Fig. 1C), suggesting a strong

218 barrier to homologous recombination between the two clades. The overall consistent branching

219 pattern between the coancestry populations and the phylogenetic groups indicates that clade-M

220 and clade-S diverged into two species. The decreased gene flow between clades is also supported

221 by decreased $\rho / \theta$ ratio and $\mathrm{r} / \mathrm{m}$ ratio between the two clades compared to those within clades

222 (Supplementary Text 2.1, Table S1). Besides, $\mathrm{F}_{\mathrm{st}}$, a measurement of population differentiation,

223 was estimated across the core genome, and the genome-wide high $\mathrm{F}_{\mathrm{st}}$ values $(\geq 0.5)$ revealed that

224 the speciation between the two clades is approaching completion (Fig. S2, Supplementary Text

$2252.1)$.

226 Recombination at the core genomic regions with distant lineages can be a key mechanism

227 driving the differentiation of the Roseobacter population [44]. Such allelic replacements are

228 expected to leave a strong signature of nucleotide substitution rate at the largely neutral

229 synonymous sites $\left(d_{S}\right)$ in the affected genes [54-56], manifested as unusually large $d_{S}$ values at 
230 those loci for between-clade comparisons. We have recently developed a pipeline to pull out

231 those loci through a clustering of the $d_{S}$ values between all possible pairs of genomes and across

232 all shared single-copy gene families (Fig. S3, Supplementary Text 1.6) [48]. This procedure led

233 to the identification of an outlier cluster consisting of 167 single-copy gene families each

234 showing unusually large between-clade $d_{S}$ values (Table S2, Fig. 2). Novel allele replacement at

235 these loci likely occurred at the last common ancestor (LCA) of either clade-M (marked with a

236 blue triangle in Fig. 1A) or clade-S (marked with a pink triangle in Fig. 1A), and the inferred

237 evolutionary history of allelic replacements was discussed in the following sections (Fig. S4, Fig.

238 S5-S11).

239

240 Differentiation of the Ruegeria population along coral compartments at the accessory genome

241 The above analyses demonstrated the genetic separation of clade-M from clade-S across

242 the core genome. We further investigated whether population differentiation also occurred in the

243 accessory genome (i.e., genes not shared by all members of the clade-M and clade-S). The

244 clustering dendrogram based on the gene presence/absence pattern (Fig. 1D) showed that the

245 similarity of accessory gene content was higher within each clade than that between the two

246 clades. The congruent branching pattern between the gene content dendrogram (Fig. 1D) and the

247 core gene-based phylogenomic tree (Fig. 1A) suggests that population differentiation has

248 occurred at the accessory genome. Among the 3,202 accessory gene families, 194 and 157

249 families are universally and exclusively present in clade-M and clade-S members, respectively.

250 Using a relaxed definition of "clade-specific gene families" where genes are present in at least

251 two-thirds of the strains in one clade but present in no more than one-third of the strains in the

252 other clade, we found 536 and 365 gene families specific to the clade-M and clade-S (Table S3, 
253 Table S4 and Fig. 2), respectively, suggesting that those two clades have diversified in many

254 clade-specific functions.

Guided by the above principles, we inferred the evolutionary history of the clade-specific

256 gene families. For the 536 clade-M specific genes, 224 and 64 gene families were inferred to be

257 acquired at the LCA of clade-M and lost at the LCA of clade-S, respectively (Table S3). Since

258 the basal position of the clade-M is occupied by a seawater strain (Fig. 1A), we further inferred

259 that 122 additional gene family gains occurred at the LCA (marked with a red star in Fig. 1A) of

260 the remaining five members which are exclusively mucus-associated (Table S3). For the 365

261 clade-S specific genes, 233 and 97 gene families were inferred to be gained at the LCA of

262 clade-S and lost at the LCA of clade-M, respectively (Table S3). Thus, both gene gains and

263 losses likely played an important role in differentiating the accessory genome. Among the 901

264 clade-specific gene families, 126 are associated with genomic islands (GIs) (Fig. 2), suggesting

265 that GIs likely facilitated population differentiation. In the following sections, we elaborated on

266 some clade-specific gene functions that may be of particular interest in terms of their potential

267 roles in facilitating population differentiation.

\section{Metabolic adaptation of the mucus population to the eutrophic mucus niche}

270 Utilization of methylamine-related osmolytes

271 A series of chemically related methylamines, such as glycine betaine (GBT),

272 choline-O-sulfate (COS), glycerophosphocholine (GPC), choline and sarcosine, are compatible

273 solutes and detected as cellular components of cnidarians including corals $[57,58]$. Among these,

274 GBT is the most prevalent osmolyte in corals [59]. GBT varies from 19 to $130 \mathrm{mmol} / \mathrm{L}$ in

275 concentration among different coral species [58] and represents up to $90 \%$ of detected 
compatible solutes or $16 \%$ of the total $\mathrm{N}$ storage in some GBT-rich corals $[10,59]$. Another two

277 methylamines, trimethylamine N-oxide (TMAO) and dimethylglycine (DMG), are also marine

278 osmolytes that can be transformed from choline and GBT (Fig. 3) [9], but have not been reported

279 in coral studies.

280 Many of the clade-M specific genes are likely involved in the utilization of these

281 methylamines. The methylamines may be transported into the bacterial cytoplasm through

282 multiple transporters. For example, GBT may be taken up through the ABC transporter ProVWX

283 [60]. The GBT precursors, GPC and choline, may be assimilated through the ABC transporter

284 UgpABCE [61] and the BCCT transporter BetT [62], respectively. Clade-M members

285 specifically contain an extra copy of all three transporters (Table S3, Fig. 3). After transportation

286 into the cytoplasm, the metabolic pathways for GBT, GPC and choline are largely overlapped

287 (Fig. 3). Choline is either incorporated into phosphatidylcholine (PC) or subjected to

288 demethylative degradation $[63,64]$. For the former, the key gene encoding choline kinase (ckil,

289 Table S3) for assimilation is specific to clade-M. For the latter, the choline is oxidized to GBT

290 aerobically before demethylation (Fig. 3). The key genes including the choline dehydrogenase

291 (betA, Table S3) and betaine aldehyde dehydrogenase (betB, Table S3) are part of the clade-M

292 specific genes (Fig. 3).

293 The model strain of the Roseobacter group, Ruegeria pomeroyi DSS-3, was previously

294 shown to degrade GBT and produce intermediate metabolites including DMG, sarcosine and

295 glycine sequentially [64]. This canonical pathway allows roseobacters to use GBT as a C, N and

296 energy source (Fig. 3, the right-hand pathway located downstream of GBT) [64]. It starts from

297 the demethylation of GBT to DMG catalyzed by betaine-homocysteine methyltransferase (bhmt)

298 (Fig. 3) [64]. In the present Ruegeria population, however, a truncated bhmt gene 
(HKCCD4315_03106) which lacks the vitamin $\mathrm{B}_{12}$-binding domain is present in the core

300 genome [65]. This truncated bhmt was also identified in the Roseobacter group member

301 Phaeobacter inhihens (previously known as Phaeobacter gallaeciensis), and was considered

302 nonfunctional for this reaction as the bacterium poorly used GBT as a C source [66]. Likewise,

303 none of the six isolates assayed here were able to thrive on choline or GBT as a sole C and N

304 source (Fig. 4A-2, 4A-3) or as a sole C source (Fig. 4B-3, 4B-5). When provided with the

305 intermediate metabolites downstream of GBT, such as DMG (Fig. 4A-4) and sarcosine (Fig.

306 4A-5), these isolates thrived, further supporting that the utilization of GBT is blocked at the step

307 catalyzed by BHMT. Specifically, DMG is demethylated to sarcosine through dimethylglycine

308 dehydrogenase ( $d m g d h)$, and sarcosine can be demethylated to glycine through either sarcosine

309 dehydrogenase (sardh) or sarcosine oxidase (soxABDG). Sarcosine also connects to another coral

310 osmolyte creatine [57]; while creatine is not a downstream metabolite of GBT, it is hydrolyzed to

311 sarcosine and urea through creatinase ( $\mathrm{cre}$ ) and the products follow the sarcosine demethylation

312 pathway and ureolysis (ureABCDEF), respectively (Fig. 3).

313 On the other hand, all assayed isolates can use choline (Fig. 4B-4) and GBT (Fig. 4B-6)

314 as sole $\mathrm{N}$ sources, suggesting that they may acquire $\mathrm{N}$ from choline and GBT through an

315 alternative pathway. One possibility is that GBT is initially transformed to trimethylamine (TMA)

316 which can be used as a sole N source (Fig. 4B-8). This reaction was experimentally demonstrated

317 in a metabolomics study on intestinal microbiota [67], but the genetic basis has not been known

318 (Fig. 3). TMA can be sequentially demethylated to dimethylamine (DMA) and

319 monomethylamine (MMA) through dimethylamine/trimethylamine dehydrogenase ( $d m d$-tmd)

320 [68]. Then MMA is further oxidized to release ammonium via a pathway comprising the

321 gamma-glutamylmethylamide synthetase ( $g m a S), \mathrm{N}$-methylglutamate synthase ( $m g s A B C$ ), and 
$322 \mathrm{~N}$-methylglutamate dehydrogenase $(\operatorname{mgdABCD})[69]$. TMAO is another methylamine osmolyte

323 that is chemically related to choline. It can be reduced to TMA through either anaerobic dimethyl

324 sulfoxide/trimethylamine oxide reductase $(\operatorname{dms} A B C)$ [70] or aerobic methionine sulfoxide

325 reductase (yed $Y Z$ ) [71], and then enters the TMA demethylation pathway (Fig. 3).

While the above-mentioned genes involved in methylamine-related osmolytes are

327 common to all members of the Ruegeria population, many of them have at least one additional

328 copy specific to the clade-M members. The latter includes ugpABCE for GPC uptake, betABT

329 and ckil for choline acquisition and catabolism, proVWX for GBT uptake, cre for converting

330 creatine to sarcosine, sardh and soxABDG for sarcosine demethylation, and $g m a S$ and $m g s A B C$

331 for MMA oxidation (Fig. 3). The majority of these genes were inferred to be acquired at the LCA

332 of clade-M (12 out of 23) or lost at LCA of clade-S (four out of 23), while the remaining genes

333 were acquired at the LCA of the five clade-M mucus members (seven out of 23) (Table S3).

334 Among the core gene copies, the ugpABE, betA, $d m g d h$, and $d m s A B C$ were subjected to novel

335 allelic replacements, though different component genes in each gene cluster went through

336 different evolutionary history of homologous recombination (Fig. 3, Table S5). In line with these

337 between-clade differences at the genetic level, the clade-M exhibited a significantly higher

338 ability than clade-S to utilize the abundant osmolytes in corals, such as using choline $(p<0.05$,

339 One-way Repeated-Measures ANOVA; the same test used below unless stated otherwise; Fig.

340 4B-4) and GBT (Fig. 4B-6) as a sole N source, and using sarcosine and creatine as a sole N and

341 C source (Fig. 4A-5 and 4A-8). While the osmolytes less common in corals (e.g. DMG, TMA

342 and TMAO) were also used by the Ruegeria population, no significant differences between

343 clade-M and clade-S were observed (Fig. 4A-4, 4B-8 and 4B-10). Detailed results of the

344 physiological assays can be found in Supplementary Text 2.2. 
To sum up, the above-mentioned genes were related to the metabolism of

346 methylamine-related osmolytes, and most of them underwent either gene copy number variation

347 or novel allelic replacements at the LCA of clade-M or clade-S. We therefore propose that the

348 acquisition of genetic traits in utilizing methylamine-related coral osmolytes contributed to the

349 diversification of clade-M from clade-S.

351 Utilization of other osmolytes in coral

Here, we highlighted three additional coral osmolytes that differentiated the clade-M

353 from the clade-S: L-proline, DMSP and taurine [10, 58, 72]. L-proline is a potential substrate of

354 the transporters ProVWX and BetT with relatively lower affinity compared to choline and GBT,

355 respectively $[60,62]$. This is supported by the growth assay (Supplementary Text 2.2), in which

356 the clade-M members showed significantly higher growth yields and growth rates in utilizing

357 L-proline than the clade-S members (Fig. 4A-9). DMSP can be degraded via either

358 demethylation or cleavage pathway, yielding methanethiol (MeSH) and dimethyl sulfide (DMS)

359 as the final products, respectively [73]. The demethylation pathway is catalyzed by $d m d A B C D$,

360 which was found exclusively in clade-M members and inferred to be acquired at the LCA of

361 clade-M (Table S3). The cleavage pathway is mediated by a number of non-homologous genes,

362 among which $d d d P$ (Table S3, Fig. 2) is exclusively present in the plasmid of clade-M members

363 and $d d d D$ (Table S5) is part of the outlier core genes showing unusually large between-clade $d_{S}$

364 values, though the donor of the novel $d d d D$ allele is unclear based on our phylogenetic analysis

365 (Fig. S4 versus Fig. S6). Using DMSP as a sole C source, the clade-M members accumulated a

366 higher growth yield than the clade-S members (Fig. 4B-2). In terms of taurine, the genes

367 encoding the taurine transporter $(\operatorname{tau} A B C)$ are part of the outlier core genes that show unusually 
368 large between-clade $d_{S}$ values (Table S5). Phylogenetic analyses showed an independent

369 evolutionary history of the three-component genes (Fig. S4 versus Fig. S7). For example, the

370 tauC phylogeny suggests that the LCA of the five clade-M mucus members (marked with a red

371 star in Fig. 1A) was subjected to divergent allele replacement, whereas the tauA phylogeny

372 indicates that the seawater strain (HKCCD6109) and clade-S were the recipients of novel alleles.

373 Notably, a second copy of the $\operatorname{tau} A B C$ genes was found in four out of the six clade-M members

374 (Table S3). These genetic differences are again supported by the growth assays, in which the

375 clade-M members showed significantly higher growth yields in the utilization of taurine as a sole

$376 \mathrm{C}$ and N source compared to the clade-S members (Fig. 4A-10). Most of the above-mentioned

377 genes were acquired at the LCA of clade-M (7 out of 11) and the remaining four genes were

378 acquired at the LCA of four clade-M members (Table S3), further supporting the utilization of

379 coral osmolytes may be the ecological adaption of clade-M bacteria.

381 mucus related substrates (i.e. carbohydrates and aromatic compounds, Supplementary Text 2.3),

382 as well as genes involved in quorum sensing (QS) system and biofilm formation (Supplementary

383 Text 2.4), which may further facilitate the clade-M members to adapt to the nutrient-rich and

384 densely-populated coral mucus habitat.

Metabolic adaptation of skeleton population to anoxic and low-energy skeleton niche

387 Energy conservation in the periodically anoxic skeleton

388 Members of the Roseobacter group commonly conserve energy through the oxidation of

389 inorganic reduced sulfur and carbon monoxide through the sox and cox gene clusters,

390 respectively [74]. We identified a complete sox gene cluster in all members of the clade-S but in 
only one strain (HKCCD4884) of the clade-M (Table S4), and inferred that this sox gene cluster

392 was lost at the LCA of clade-M. Additionally, two cox gene clusters encoding the form I

393 (HKCCD4315_03676-03684) and form II (HKCCD4315_03987-03970) carbon monoxide

394 dehydrogenase ( codh) are shared by both clades. Among these, the form I cox gene cluster (Table

395 S5), which is indispensable for carbon monoxide oxidation [75], showed unusually large

396 between-clade $d_{S}$ values, suggesting the different genomic origins of carbon monoxide oxidation

397 between the two clades. The phylogenetic trees of the component genes comprising the form I

398 cox cluster consistently showed that the LCA of clade-S was subjected to divergent allele

399 replacements at the cox gene cluster (Fig. S4 versus Fig. S8). Together with the presence of sox

400 gene cluster, the clade-S members appear to use more versatile energy conservation strategies to

401 adapt to the energy-limited coral skeleton niches.

402 The dissolved oxygen produced by the photosynthesis of symbiotic and endolithic algae

403 during daytime is continuously consumed by the holobiont members through respiration [15],

404 making the skeleton a diurnally anoxic environment [76]. Consistently, the clade-S acquired a

405 gene cluster ( $\mathrm{rdABCD}$ and $t \mathrm{tdAB}$, Table S4) at LCA which likely enables the skeleton-associated

406 strains to respire fumarate and L-tartrate under anoxic conditions. Besides, a gene cluster

407 allowing for anaerobic respiration of dimethyl sulfoxide (DMSO) and TMAO showed an

408 unusually large $d_{S}$ value (Supplementary Text 2.5, Fig. S9). These compounds could serve as the

409 terminal electron acceptors for energy conservation in the diurnally anoxic skeleton [77].

411 Motility

412 Bacteria can be motile in multiple ways, among which the swimming motility enables

413 bacteria to move in the liquids, while swarming and twitching allow the bacteria to be motile on 
414 solid surfaces (e.g., coral skeleton) [78]. In the Roseobacter group, the swimming motility is

415 widely observed, but swarming motility has not been reported [79, 80]. Both swimming and

416 swarming may be conferred by any of the three evolutionarily related flagellar gene clusters

417 (FGCs), termed fla1, fla2 and fla3 [79, 80]. The putative twitching motility resulting in a

418 dendritic-shaped phenotype was observed in seven Roseobacter species, but the genetic basis has

419 not been clear [79].

All clade-S members except for HKCCD7318 carry the complete set of flal consisting of

42136 genes (Fig. 5A). In contrast, two DNA segments encompassing 17 continuous genes and part

422 of the flgI gene (Table S4) are missing from the clade-M members (Fig. 5A), and they were

423 inferred to be lost at the LCA of clade-M. These lost genes are involved in the assembly of

424 multiple components of the flagellum, including components of type III secretion system (flhA,

$425 f l h B, f l i Q$, and $f l i R)$, P- and L-rings $(f l g A$ and $f l g H)$, motor proteins ( $\operatorname{mot} B)$, basal body proteins

$426(f \lg B, f \lg C, f \lg G$ and $f l i I)$, hook $(f l g E, f l g K, f l g L$ and $f l i E)$ and flagellum-specific ATP synthase

427 (fliI). The strain HKCCD7318, an exception of the clade-S members, also carries a truncated fla1

428 cluster, but encompasses five more genes encoding components of type III secretion system and

429 basal body compared to the clade-M members (Table S4, Fig. 5A). Our experimental assays

430 showed that the clade-S members with a complete set of flal (HKCCD5849 and HKCCD7319)

431 displayed larger swimming circles than the clade-M members and the HKCCD7318 which

432 carried a truncated flal cluster (Fig. 5B). No swarming or twitching motility was observed in all

433 tested members (Fig. 5B). Previous studies on Rhodobacter sphaeroides showed that the

434 expression of the flal cluster is positively regulated under anaerobic conditions which could be

435 part of the aerotaxic response of this bacterium to oxygen or alternative electron acceptors (e.g.,

436 DMSO and TMAO) in response to the anoxic condition [81]. This helps explain why the 
437 complete flal in some clade-S members is maintained. As mentioned above, the coral skeleton

438 becomes periodically anoxic, and the maintained motility may facilitate scavenging oxygen and

439 alternative electron acceptors in the skeleton with compacted aragonites.

441 Possible role of the urea remineralization potential

442 Coral reef ecosystem is generally poor in $\mathrm{N}$ [82]. Urea, mainly from the excretion of

443 zooplankton, fish and other animals, is an important source of $\mathrm{N}[82,83]$ for the coral host [84,

444 85], Symbiodiniaceae [85] and bacteria [86] in the coral holobiont. Urea is hydrolyzed to

445 ammonium and inorganic carbon by urease and results in the rise of $\mathrm{pH}$ and carbonate

446 concentration [87]. Ureolysis by bacteria is known to facilitate calcification by increasing $\mathrm{pH}$ and

447 supplying carbonate in environments such as soil, estuarine, coastal seawater, animal rumen and

448 gut [88-91]. In the coral skeleton, the calcification process can also be enhanced by ureolysis [92,

449 93].]

450 A gene cluster encoding the urea transporter (urtABCDE, Table S4) is specific to the

451 clade-S, and four ( $u r t A B C D)$ out of the five subunits were likely lost at the LCA of clade-M

452 (Table S4). Besides, the genes (ureABCDEF, Table S5) encoding the urease for urea hydrolysis

453 are part of the core genes showing unusually large between-clade $d_{S}$ values. Phylogenetic

454 analyses suggested that either clade-S or clade-M acquired alleles from a different genetic origin,

455 depending on the component gene (Fig. S4 versus Fig. S10). These results suggest that the

456 clade-M and clade-S have differentiated on the urea decomposition, and the clade-S may show a

457 higher potential to take advantage of this function as it kept the transporter for urea uptake. In

458 that case, the conservation of genetic traits in urea mineralization by skeleton-associated

459 members might be beneficial for the calcification of the coral host. 
461 poorly on urea under the tested conditions and did not exhibit differential growths (Fig. 4A-11,

462 4B-11 and 4B-12). We argue that generating N source is not the primary purpose of the Ruegeria

463 population to use urea. This is because urea may not be a preferred $\mathrm{N}$ source of bacteria due to

464 the high energy cost in using it [94], especially when the coral skeleton is enriched with

465 dissolved inorganic nitrogen with its concentration exceeding that in ambient seawater by a

466 factor of 10 [95]. Besides using urea as $\mathrm{N}$ source [96], bacteria also benefit from ureolysis for

467 survival in acidic environments [97] and chemotaxis to the host [98], which are induced by acid

468 exposure [97] and enrichment of nickel (Ni) [99], respectively. However, the environmental

469 inducers of urease expression (i.e., acidic $\mathrm{pH}$ and high level of $\mathrm{Ni}$ ) were not screened in the

470 present study, which might explain the overall poor utilization of urea by the Ruegeria

471 population and the absence of between-clade differences. We hypothesize that ureolysis in the

472 skeleton may be a conditionally expressed function in response to undetected environmental

473 inducers, which may increase the calcification efficiency of coral host meanwhile. Future

474 experiments are needed to validate this hypothesis.

475

The role of different coral compartments in driving the Ruegeria population evolution

The metabolic differences between the clade-M and clade-S provide a wealth of evidence

478 that the diversification of the two clades is likely driven by heterogeneous conditions associated

479 with different coral compartments. However, the splitting time of the two clades was estimated to

480 be 7.52 million years ago, indicating that the split of clade-M and clade-S far predated the birth

481 of their host coral which likely emerged several decades to a century ago [100]. The long

482 evolutionary time thus leaves the origin of the clade-M and clade-S an unanswered question: did 
483 they evolve de novo on coral, or had they already occurred in other environments before their

484 colonization on the different compartments of the coral host?

In the former case, different compartments is the direct force driving speciation. The

486 bacteria have a chance to undergo a long evolution time on corals even beyond the longevity of

487 coral, because bacteria could be transmitted vertically between coral generations (Fig. S11) [101].

488 In this model, the LCA of the clade-M and clade-S colonized the ancient coral host, then the

489 clade-M and clade-S diverged in situ through the acquisition or loss of metabolic traits and

490 thrived in their preferred coral compartments along with the growth cycle of the coral host. The

491 predominant bacterial clade in one compartment might still be able to survive in another as they

492 are spatially close, but with a relatively lower abundance due to compartment-specific selection

493 for its own inhabitants. Next, members of both clades have a chance to be transmitted together

494 with the coral gametes and the planula, and thrive in their optimum compartments after the coral

495 recruit is settled and starts to secret mucus and develop the skeleton.

496 If the second hypothesis is true (Fig. S11), distinct coral compartments may not be the

497 initial force driving the speciation between the clade-M and clade-S. Instead, the two clades

498 might have already diverged in other environments and repeatedly colonized on different

499 compartments of the coral host throughout their evolutionary processes. The environmental

500 heterogeneity of different coral compartments might impose distinct selective forces on the

501 localized bacteria and enrich the best-adapted clade members, and this in turn may accelerate the

502 diversification of the two clades.

503 To help resolve these competing hypotheses, we included additional 24 strains that are

504 closely related to members of the Ruegeria population. Among these, 22 co-occurred with the

505 coral-associated Ruegeria population in the Hong Kong coastal ecosystems, but they were 
506 isolated from a variety of ecological niches including brown algal ecosystem niches (e.g.

507 seawater, sediments and algal tissue), mangrove rhizosphere, coastal seawater, intertidal

508 sediments and another batch of the P. acuta coral sample (Table S6). According to the updated

509 phylogenomic tree (Fig. 5C), the newly added strains expanded all three major clades of the

510 Ruegeria population (Fig. 5C). Members in the expanded clade-M (hereafter "exp-clade-M")

511 were equally partitioned into two subclades, in which subclade-M1 is dominated by the

512 mucus-associated members (equivalent to the clade-M in Fig. 1A) and subclade-M2 consists of

513 members largely from non-coral niches (Fig. 5C). This pattern prevented us from inferring the

514 ancestral habitat of the LCA of exp-clade-M. Members in the expanded clade-S (hereafter

515 “exp-clade-S”) were unequally grouped into two subclades, with the singleton subclade-S2

516 derived from seawater and the subclade-S1 composed of phylogenetically mixed strains

517 inhabiting the coral skeleton and other non-coral niches (Fig. 5C). Thus, we cannot conclusively

518 determine the habitat of the LCA of the exp-clade-S. In the case of the expanded outgroup clade,

519 the coral-associated strains are embedded in the early-branching lineages that are not derived

520 from corals (Fig. 5C), suggesting that the ancestral habitat of the LCA of this expand outgroup

521 clade was not related to coral. When these three clades were viewed collectively, together with

522 the deeply-branching singleton clade represented by a seawater strain Ruegeria sp. 6PALISEP08

523 located at the middle of the tree (Fig. 5C), the available isolates' habitat information and

524 phylogenetic structure are more consistent with the hypothesis that the original skeleton clade,

525 mucus clade, and outgroup clade (Fig. 5C) each independently evolved from ancestors

526 colonizing non-coral habitats.

527 Since the cladogenesis event between the original clade-M and clade-S was likely

528 completed in habitats other than the coral compartments, some between-clade differences of 
metabolic potential could have existed before ancestral lineages independently transited to coral

530 mucus and skeleton. We therefore sought evidence that allows singling out the metabolic

531 differences imposed by different coral compartments from those by non-coral environments. In

532 brief, we screened the gene families that show clade-M (or clade-S) specific distribution but are

533 not prevalent in exp-clade-M (or exp-clade-S), because the evolution of these genes is more

534 likely driven by coral compartments. We then inferred the evolutionary history of the eligible

535 gene families along the genome-based phylogeny of the 44 strains (Fig. 5C).

537 clade-M discussed above were acquired at the LCA of the subclade-M1 in the expanded

538 phylogeny (Table S7). These genes include ugpABCE for GPC uptake, betAB for choline

539 oxidation, gmaS and $m g s A B C$ for MMA oxidation, cre for creatine degradation, $\operatorname{tau} A B C$ for

540 taurine uptake, and $d d d P$ for DMSP lysis. This is evidence that the coral mucus environment

541 represents an important selective force shaping the genome of mucus-associated bacteria. This

542 expanded analysis, however, rendered the evolution of the remaining osmolyte degradation genes

543 specific to clade-M uncoupled from the coral mucus habitat, since these genes were prevalent

544 among the exp-clade-M members and were largely acquired at the LCA of the exp-clade-M

545 (Table S7). Similarly, the expanded analysis uncorrelated the evolution of urea transporter genes,

546 sulfur oxidation genes, and fumarate and L-tartrate respiration genes to the coral skeleton habitat,

547 since these genes were commonly found in the exp-clade-S members and were either acquired at

548 the LCA of exp-clade-S or lost at the LCA of exp-clade-M (Table S8). In the case of the type I

549 flagellar gene cluster (flal) comprising 18 genes, it is difficult to infer its evolutionary history

550 based on the gene presence and absence pattern. This is because while a few members in the

551 exp-clade-S including most skeleton-associated members possess a complete set of the genes, the 
552 basal branch of this clade and all members of exp-clade-M each contain a subset of the genes

553 (Fig. 5). In the strains carrying an incomplete flal cluster (Fig. 5C), the genes adjacent to the

554 missing part of the flal were mostly pseudogenized (Fig. 5A). Because pseudogenization is

555 unequivocal genetic evidence of ongoing gene loss [50], the missing part of flal is more likely a

556 result of gene loss (Table S8). Therefore, the conservation of a complete flal cluster in most

557 skeleton-associated members indicates that the coral skeleton may act as a selective force to

558 maintain flal in the clade-S.

560 Limited migration of a genetically uniform Rhodobacteraceae population across coral

$561 \quad$ compartments

562 For the Ruegeria population, we provided evidence that different compartments of the

563 coral host likely act as distinct selective pressures that drive the evolution and adaptation of

564 bacterial genomes. Given a considerable amount of genetic diversity harbored in that population,

565 however, it remains unknown whether different compartments of the coral host also act as a

566 physical barrier of gene flow among bacteria colonizing different compartments. We next

567 investigated this question by using a genetically uniform Rhodobacteraceae population, which is

568 composed of 214 isolates sampled from four different coral individuals of the same coral species

$569 P$. acuta (Table S6), each collected from a different location in Hong Kong (Fig. S1). This new

570 population represents a distinct lineage from all known Roseobacter group members (Fig. S4),

571 showing identity of $97.7 \%$ at the $16 \mathrm{~S}$ rRNA gene to its closest relative, Rhodobacteraceae

572 bacterium MA-7-27 (GenBank Assembly Accession Number: GCA_003688285.1). Members of

573 this population share identical 16S rRNA genes, show the pairwise ANI at $99.99 \%$ (Table 1),

574 and differ by only 43 SNPs across around 4.39 Mbp core genome sequences shared by the 214 
575 strains according to kSNP3 [102] (Table 1). Phylogenomic construction based on these SNPs

576 showed that the isolates are not clustered according to the coral compartments or the sampling

577 locations (Fig. S13). The extremely high genetic identity among isolates indicates a very recent

578 origin of this population within the past several hundreds of years [130 and 94 years ago for the

579 Wong Wan Chau (WWC) and Ngo Mei Chau (NMC) subpopulations, respectively.

580 Supplementary text 1.11], which likely spanned up to a few generations of the coral animals in

581 Hong Kong waters [100] and thus provided a unique opportunity to test whether dispersal

582 limitation may have a role in driving population differentiation.

583 The Slatkin-Maddison test [51] is designed to measure the extent of

584 compartmentalization according to the number of migrations producing the observed

585 phylogenetic placement (Supplementary Text 1.10). By assuming that the common ancestor of

586 closely related strains occupied a single compartment, a migration event is inferred when a

587 closely related strain is found in a different compartment. The small probability that the

588 estimated number of migrations from the real data is greater than the expected number of

589 migrations calculated from randomly generated population structures by permutating the strains

590 across the phylogenomic tree reflects the high degree of compartmentalization. The WWC

591 subpopulation (Fig. 6A) and the NMC subpopulation (Fig. 6B) each consist of two or more

592 compartments, and thus are qualified for the Slatkin-Maddison test. This test showed that in both

593 subpopulations fewer migrations were inferred than expected by chance (29 migrations in the

594 WWC subpopulation, $p<0.01$, Fig. 6C; 12 migrations in the NMC subpopulation, $p<0.001$, Fig.

595 6D), indicating that members from distinct compartments of the same coral individual are

596 compartmentalized and that microbial population differentiation along coral compartments likely

597 started from limited migration between compartments. 


\section{Concluding remarks}

600 Coral hosts harbor multiple compartments that differ in many physicochemical properties,

601 including nutrient quality and quantity, energy sources and redox levels among others. Here, we

602 provided the first evidence that this heterogeneity drives the population evolution of bacterial

603 residents. Of the two Roseobacter group populations examined here, one (the Rhodobacteraceae

604 population) differs at only a few SNP sites across the whole genomes but has already shown

605 dispersal limitation across the different compartments, whereas the other (the Ruegeria

606 population) has already diverged into distinct species varying in multiple functional traits. For

607 the latter, gene gains and losses at the accessory genome along with novel allele replacements at

608 the core genome are likely the underlying processes driving their differentiation. While the

609 clade-M acquired novel genes to make use of mucus-related compounds (e.g., methylamines and

610 other coral osmolytes, carbohydrates and aromatics) and to enable them to compete well with

611 other bacteria in the densely populated coral mucus; clade-S maintained metabolic traits lost by

612 clade-M, such as oxidation of inorganic sulfur and carbon monoxide, motility, and potential urea

613 decomposition, and also acquired new traits to distinguish themselves from the outgroup, which

614 are of vital importance to survive in skeletons. Although some traits may have diversified before

615 the colonization of the coral host, the differentiation in a few important traits including motility

616 and utilization of mucus-derived compounds indicates skeleton and mucus environments may be

617 important selective forces shaping the Ruegeria population evolution.

618 Previous coral microbiome studies successfully characterized the prevalence of

619 Rhodobacteraceae members in coral environments at the community structure level, but the role

620 of each lineage on corals, and more specifically, on different coral compartments has not been 
621 appreciated. Population genomics is a powerful tool to trace how specific lineages evolve along

622 different coral compartments, and brings us the knowledge of how bacteria interact with coral

623 through neutral processes due to dispersal limitation and adaptive choices of local groups. By

624 taking advantage of population genomics, our analysis provides the first insights that evolution

625 of the ecologically relevant Ruegeria population is driven by the specific environmental factors

626 of distinct coral compartments. Some of the local adaptation strategies are win-win choices for

627 the bacteria and coral host. For example, clade-M members may remove some potentially toxic

628 aromatics trapped in the coral mucus, and clade-S members possess genetic potential to

629 decompose urea, which might confer benefits to the coral host by promoting calcification of the

630 skeleton when the environment acidifies. These predictions, if confirmed, would be the building

631 blocks to further understand the whole nature of the bacteria-coral association and would help to

632 improve our strategies in coral conservation by more carefully considering the role of bacteria in

633 maintaining the health of coral holobiont.

\section{Acknowledgments}

636 We thank Ryan Ho-Leung Tsang for the coral sample collection, Tsz-Yan Ng for guiding

637 coral sample processing, Xiao Chu and Shuangfei Zhang for their help on bacteria cultivation,

638 Hao Zhang and Minglei Ren for their help in data analysis, and Xinqin Lin for her advice in

639 experimental design. We thank Xiao Chu, Minglei Ren and Zhichao Zhou for providing isolates

640 from the brown algae ecosystem, sediments and mangrove ecosystem. This work was supported

641 by the Shenzhen Science and Technology Committee (JCYJ20180508161811899), the Hong

642 Kong Environment and Conservation Fund (15/2016), the Hong Kong Research Grants Council 
643 General Research Fund (14163917), and the Hong Kong Research Grants Council Area of

644 Excellence Scheme (AoE/M-403/16).

645

\section{Conflict of Interest}

647 The authors declare no competing commercial interests concerning the submitted work.

648

\section{Data Availability}

650 The assembled genomic sequences and raw reads of the coral-associated Ruegeria

651 population, the seven Ruegeria isolates from non-coral marine habitats, and the coral-associated

652 Rhodobacteraceae population are made publicly available at NCBI under GenBank assembly

653 accession number PRJNA596594, PRJNA682389 and PRJNA596592, respectively. 


\section{References}

6551 Bourne DG, Morrow KM, Webster NS. Insights into the coral microbiome: underpinning

656 the health and resilience of reef ecosystems. Annu Rev Microbiol. 2016; 70: 317-340.

6572 Ainsworth TD, Thurber RV, Gates RD. The future of coral reefs: a microbial perspective.

658 Trends Ecol Evol. 2010; 25: 233-240.

6593 Huettel M, Wild C, Gonelli S. Mucus trap in coral reefs: formation and temporal

660 evolution of particle aggregates caused by coral mucus. Mar Ecol Prog Ser. 2006; 307 :

$661 \quad 69-84$.

6624 Coffroth M. Mucous sheet formation on poritid corals: an evaluation of coral mucus as a

663 nutrient source on reefs. Mar Biol. 1990; 105: 39-49.

6645 Brown BE, Bythell JC. Perspectives on mucus secretion in reef corals. Mar Ecol Prog Ser.

665 2005; 296: 291-309.

6666 Sweet M, Croquer A, Bythell J. Bacterial assemblages differ between compartments

667 within the coral holobiont. Coral Reefs. 2011; 30: 39-52.

6687 Yancey PH. Organic osmolytes as compatible, metabolic and counteracting

669 cytoprotectants in high osmolarity and other stresses. J Exp Biol. 2005; 208: 2819-2830.

6708 Burg MB, Ferraris JD. Intracellular organic osmolytes: Function and regulation. J Biol

671

672 Chem. 2008; 283: 7309-7313.

673

9 Singh LR, Dar TA (eds). Cellular Osmolytes: From Chaperoning Protein Folding to Clinical Perspectives. 1st edn. (Springer Nature Singapore Pte Ltd, Singapore, 2017).

675

11 Mayfield AB, Gates RD. Osmoregulation in anthozoan - dinoflagellate symbiosis. Comp Biochem Physiol A. 2007; 147: 1-10.

12 Rublee PA, Lasker HR, Gottfried M, Roman MR. Production and bacterial colonization of mucus from the soft coral Briarium asbestinum. Bull Mar Sci. 1980; 30: 888-893. Wild C, Woyt H, Huettel M. Influence of coral mucus on nutrient fluxes in carbonate sands. Mar Ecol Prog Ser. 2005; 287: 87-98.

14 Coles SL, Strathmann R. Observations on coral mucus "flocs" and their potential trophic significance. Limnol Oceanogr. 1973; 18: 673-678.

15 Pernice M, Raina J-B, Rädecker N, Cárdenas A, Pogoreutz C, Voolstra CR. Down to the bone: the role of overlooked endolithic microbiomes in reef coral health. ISME J. 2020;

$68816 \quad$ Falini G, Fermani S, Goffredo S. Coral biomineralization: A focus on intra-skeletal organic matrix and calcification. Semin Cell Dev Biol. 2015; 46: 17-26. Constantz B, Weiner S. Acidic macromolecules associated with the mineral phase of scleractinian coral skeletons. J Exp Zool. 1988; 248: 253-258. 2005; 102: 1525-1530.

9 Sorek M, Díaz-Almeyda EM, Medina M, Levy O. Circadian clocks in symbiotic corals: the duet between Symbiodinium algae and their coral host. Mar Genomics. 2014; 14 : 47-57. 
69820 Rosenberg E, Koren O, Reshef L, Efrony R, Zilber-Rosenberg I. The role of microorganisms in coral health, disease and evolution. Nat Rev Microbiol. 2007; 5: 355-362.

21 Ritchie KB. Bacterial symbionts of corals and Symbiodinium. In: Rosenberg E, Gophna U (eds). Beneficial Microorganisms in Multicellular Life Forms. 1st edn. (Springer-Verlag Berlin Heidelberg, Berlin Heidelberg, 2012) pp 139-150.

22 Apprill A, Weber LG, Santoro AE. Distinguishing between microbial habitats unravels ecological complexity in coral microbiomes. mSystems. 2016; 1: e00143-00116. Pollock FJ, McMinds R, Smith S, Bourne DG, Willis BL, Medina M et al. Coral-associated bacteria demonstrate phylosymbiosis and cophylogeny. Nat Commun. 2018; 9: 1-13.

24 Shapiro BJ, Friedman J, Cordero OX, Preheim SP, Timberlake SC, Szabó G et al. Population genomics of early events in the ecological differentiation of bacteria. Science. 2012; 336: 48-51.

25 Youngblut ND, Wirth JS, Henriksen JR, Smith M, Simon H, Metcalf WW et al. Genomic and phenotypic differentiation among Methanosarcina mazei populations from Columbia River sediment. ISME J. 2015; 9: 2191-2205.

26 Wielgoss S, Didelot X, Chaudhuri RR, Liu X, Weedall GD, Velicer GJ et al. A barrier to homologous recombination between sympatric strains of the cooperative soil bacterium Myxococcus xanthus. ISME J. 2016; 10: 2468-2477.

27 Chase AB, Arevalo P, Brodie EL, Polz MF, Karaoz U, Martiny JB. Maintenance of sympatric and allopatric populations in free-living terrestrial bacteria. Mbio. 2019; 10: e02361-02319.

28 Huggett MJ, Apprill A. Coral microbiome database: Integration of sequences reveals high diversity and relatedness of coral-associated microbes. Environ Microbiol Rep. 2019; 11: 372-385.

29 Apprill A, Marlow HQ, Martindale MQ, Rappe MS. The onset of microbial associations in the coral Pocillopora meandrina. ISME J. 2009; 3: 685-699.

30 Epstein HE, Torda G, Munday PL, van Oppen MJH. Parental and early life stage environments drive establishment of bacterial and dinoflagellate communities in a common coral. ISME J. 2019; 13: 1635-1638.

31 Freire I, Gutner-Hoch E, Muras A, Benayahu Y, Otero A. The effect of bacteria on planula-larvae settlement and metamorphosis in the octocoral Rhytisma fulvum fulvum. PLoS One. 2019; 14: e0223214.

32 Miura N, Motone K, Takagi T, Aburaya S, Watanabe S, Aoki W et al. Ruegeria sp. strains isolated from the reef-building coral Galaxea fascicularis inhibit growth of the temperature-dependent pathogen Vibrio coralliilyticus. Mar Biotechnol. 2019; 21: 1-8. associated with lesioned and healthy Fungiidae corals. Environ Microbiol. 2013; 15: 2063-2072.

34 Sekar R, Kaczmarsky LT, Richardson LL. Microbial community composition of black band disease on the coral host Siderastrea siderea from three regions of the wider Caribbean. Mar Ecol Prog Ser. 2008; 362: 85-98. 
Casey JM, Connolly SR, Ainsworth TD. Coral transplantation triggers shift in

microbiome and promotion of coral disease associated potential pathogens. Sci Rep. 2015; 5: 11903-11903.

Tsang RHL, Ang PO. Resistance to temperature stress and Drupella corallivory may promote the dominance of Platygyra acuta in the marginal coral communities in Hong Kong. Mar Environ Res. 2019; 144: 20-27.

Tam TW, Ang Jr PO. Repeated physical disturbances and the stability of sub-tropical coral communities in Hong Kong, China. Aquat Conserv. 2008; 18: 1005-1024. Ang Jr PO, Choi LS, Choi MM, Cornish A, Fung HL, Lee MW et al. Hong Kong. In: Centre JWR (eds). Status of coral reefs of the East Asian Seas region: 2004. (Ministry of the Environment, Tokyo, 2005) pp 121-152.

752

753

39 Pujalte MJ, Lucena T, Ruvira MA, Arahal DR, Macián MC. The family

40 Emms DM, Kelly S. OrthoFinder: Phylogenetic orthology inference for comparative Rhodobacteraceae. In: Rosenberg E, DeLong EF, Lory S, Stackebrandt E, Thompson F (eds). The Prokaryotes: Alphaproteobacteria and Betaproteobacteria. 4th edn. (Springer-Verlag Berlin Heidelberg, Berlin Heidelberg, 2014) pp 439-512. genomics. Genome Biol. 2019; 20: 1-14.

41 Nguyen LT, Schmidt HA, von Haeseler A, Minh BQ. IQ-TREE: A fast and effective stochastic algorithm for estimating maximum-likelihood phylogenies. Mol Biol Evol. 2015; 32: 268-274.

42 Achtman M, Wagner M. Microbial diversity and the genetic nature of microbial species. Nat Rev Microbiol. 2008; 6: 431-440.

43 Feil EJ, Spratt BG. Recombination and the population structures of bacterial pathogens. Annual Reviews in Microbiology. 2001; 55: 561-590.

44 Wang X, Zhang Y, Ren M, Xia T, Chu X, Liu C et al. Cryptic speciation of a pelagic Roseobacter population varying at a few thousand nucleotide sites. ISME J. 2020: 1-14.

45 Lawson DJ, Hellenthal G, Myers S, Falush D. Inference of population structure using dense haplotype data. PLoS Genet. 2012; 8: e1002453.

46 Didelot X, Wilson DJ. ClonalFrameML: efficient inference of recombination in whole bacterial genomes. PLoS Comput Biol. 2015; 11: e1004041.

47 Excoffier L, Lischer HE. Arlequin suite ver 3.5: A new series of programs to perform population genetics analyses under Linux and Windows. Mol Ecol Resour. 2010; 10: 564-567.

48 Sun Y, Luo HW. Homologous recombination in core genomes facilitates marine bacterial adaptation. Appl Environ Microbiol. 2018; 84: e02545-02517.

49 Librado P, Vieira FG, Rozas J. BadiRate: Estimating family turnover rates by likelihood-based methods. Bioinformatics. 2011; 28: 279-281.

50 Chu X, Li S, Wang S, Luo D, Luo H. Gene loss through pseudogenization contributes to the ecological diversification of a generalist Roseobacter lineage. The ISME Journal (in press).

51 Slatkin M, Maddison WP. A cladistic measure of gene flow inferred from the phylogenies of alleles. Genetics. 1989; 123: 603-613. ANI analysis of $90 \mathrm{~K}$ prokaryotic genomes reveals clear species boundaries. Nat Commun. 
53 Varghese NJ, Mukherjee S, Ivanova N, Konstantinidis KT, Mavrommatis K, Kyrpides NC et al. Microbial species delineation using whole genome sequences. Nucleic Acids Res. 2015; 43: 6761-6771.

54 Hughes AL, Friedman R. Gene duplication and the properties of biological networks. J Mol Evol. 2005; 61: 758-764. Hughes AL, French JO. Homologous recombination and the pattern of nucleotide substitution in Ehrlichia ruminantium. Gene. 2007; 387: 31-37. Engel P, Stepanauskas R, Moran NA. Hidden diversity in honey bee gut symbionts detected by single-cell genomics. PLoS Genet. 2014; 10: e1004596. Lohr KE, Khattri RB, Guingab-Cagmat J, Camp EF, Merritt ME, Garrett TJ et al. Metabolomic profiles differ among unique genotypes of a threatened Caribbean coral. Sci Rep. 2019; 9: 1-11.

58 Hill R, Li C, Jones A, Gunn J, Frade P. Abundant betaines in reef-building corals and ecological indicators of a photoprotective role. Coral Reefs. 2010; 29: 869.

59 Ngugi DK, Ziegler M, Duarte CM, Voolstra CR. Genomic blueprint of glycine betaine metabolism in coral metaorganisms and their contribution to reef nitrogen budgets. iScience. 2020; 23: 101120.

60 Gowrishankar J. Nucleotide sequence of the osmoregulatory proU operon of Escherichia coli. J Bacteriol. 1989; 171: 1923-1931.

61 Chandravanshi M, Gogoi P, Kanaujia SP. Computational characterization of TTHA0379: A potential glycerophosphocholine binding protein of Ugp ATP-binding cassette transporter. Gene. 2016; 592: 260-268.

62 Ziegler C, Bremer E, Krämer R. The BCCT family of carriers: from physiology to crystal structure. Mol Microbiol. 2010; 78: 13-34.

63 Geiger O, López-Lara IM, Sohlenkamp C. Phosphatidylcholine biosynthesis and function in bacteria. Biochim Biophys Acta Mol Cell Biol Lipids. 2013; 1831: 503-513.

64 Lidbury I, Kimberley G, Scanlan DJ, Murrell JC, Chen Y. Comparative genomics and mutagenesis analyses of choline metabolism in the marine Roseobacter clade. Environ Microbiol. 2015; 17: 5048-5062.

65 Barra L, Fontenelle C, Ermel G, Trautwetter A, Walker GC, Blanco C. Interrelations between glycine betaine catabolism and methionine biosynthesis in Sinorhizobium meliloti strain 102F34. J Bacteriol. 2006; 188: 7195-7204.

66 Thole S, Kalhoefer D, Voget S, Berger M, Engelhardt T, Liesegang H et al. Phaeobacter gallaeciensis genomes from globally opposite locations reveal high similarity of adaptation to surface life. ISME J. 2012; 6: 2229-2244.

67 Wang Z, Klipfell E, Bennett BJ, Koeth R, Levison BS, DuGar B et al. Gut flora metabolism of phosphatidylcholine promotes cardiovascular disease. Nature. 2011; 472: 57-63.

68 Jones M, Talfournier F, Bobrov A, Grossmann JG, Vekshin N, Sutcliffe MJ et al. Electron transfer and conformational change in complexes of trimethylamine dehydrogenase and electron transferring flavoprotein. J Biol Chem. 2002; 277: 8457-8465.

69 Chen Y. Comparative genomics of methylated amine utilization by marine Roseobacter clade bacteria and development of functional gene markers ( $\mathrm{tmm}, \mathrm{gmaS}$ ). Environ Microbiol. 2012; 14: 2308-2322. 
83070 McNicholas PM, Chiang RC, Gunsalus RP. Anaerobic regulation of the Escherichia coli

831 dmsABC operon requires the molybdate-responsive regulator ModE. Mol Microbiol.

$832 \quad 1998 ; 27: 197-208$.

83371 Loschi L, Brokx SJ, Hills TL, Zhang G, Bertero MG, Lovering AL et al. Structural and biochemical identification of a novel bacterial oxidoreductase. J Biol Chem. 2004; 279 : 50391-50400.

72 Hillyer KE, Dias DA, Lutz A, Wilkinson SP, Roessner U, Davy SK. Metabolite profiling of symbiont and host during thermal stress and bleaching in the coral Acropora aspera. Coral Reefs. 2017; 36: 105-118.

73 Dickschat JS, Zell C, Brock NL. Pathways and substrate specificity of DMSP catabolism in marine bacteria of the Roseobacter clade. ChemBioChem. 2010; 11: 417-425. Luo H, Moran MA. Evolutionary ecology of the marine Roseobacter clade. Microbiol Mol Biol Rev. 2014; 78: 573-587.

75 Cunliffe M. Correlating carbon monoxide oxidation with cox genes in the abundant marine Roseobacter clade. ISME J. 2011; 5: 685-691.

76 Kühl M, Holst G, Larkum AW, Ralph PJ. Imaging of oxygen dynamics within the endolithic algal community of the massive coral Porites lobata. J Phycol. 2008; 44: 541-550.

77 Mccrindle SL, Kappler U, Mcewan AG. Microbial dimethylsulfoxide and trimethylamine-N-oxide respiration. Adv Microb Physiol. 2005; 50: 147-198.

78 Kearns DB. A field guide to bacterial swarming motility. Nat Rev Microbiol. 2010; 8: 634-644.

79 Bartling P, Vollmers J, Petersen J. The first world swimming championships of Roseobacters - phylogenomic insights into an exceptional motility phenotype. Syst Appl Microbiol. 2018; 41: 544-554.

80 Michael V, Frank O, Bartling P, Scheuner C, Goker M, Brinkmann H et al. Biofilm plasmids with a rhamnose operon are widely distributed determinants of the 'swim-or-stick' lifestyle in roseobacters. ISME J. 2016; 10: 2498-2513.

873 Armitage JP. Behavioural responses of bacteria to light and oxygen. Arch 1997; 168: 249-261.

82 Crandall JB, Teece MA. Urea is a dynamic pool of bioavailable nitrogen in coral reefs. Coral Reefs. 2012; 31: 207-214.

83 Wafar M, Wafar S, Devassy V. Nitrogenous nutrients and primary production in a tropical oceanic environment. Bull Mar Sci. 1986; 38: 273-284.

84 Barnes D, Crossland C. Urease activity in the staghorn coral, Acropora acuminata. Comp Biochem Physiol B. 1976; 55: 371-376.

85 Grover R, Maguer J-F, Allemand D, Ferrier-Pagès C. Urea uptake by the scleractinian coral Stylophora pistillata. J Exp Mar Biol Ecol. 2006; 332: 216-225.

86 Zhou Y, Tang K, Wang P, Wang W, Wang Y, Wang X. Identification of bacteria-derived urease in the coral gastric cavity. Sci China Earth Sci. 2020; 63: 1553-1563.

87 Krajewska B. Ureases I. Functional, catalytic and kinetic properties: A review. J Mol Catal B Enzym. 2009; 59: 9-21.

88 Cheng L, Cord-Ruwisch R. In situ soil cementation with ureolytic bacteria by surface percolation. Ecol Eng. 2012; 42: 64-72. 
87489 Cho BC, Park MG, Shim JH, Azam F. Significance of bacteria in urea dynamics in coastal surface waters. Mar Ecol Prog Ser. 1996; 142: 19-26.

87690 Jin D, Zhao SG, Zheng N, Beckers Y, Wang JQ. Urea metabolism and regulation by rumen bacterial urease in ruminants - a review. Ann Anim Sci. 2018; 18: 303-318.

87891 Collier JL, Baker KM, Bell SL. Diversity of urea-degrading microorganisms in open-ocean and estuarine planktonic communities. Environ Microbiol. 2009; 11: 3118-3131.

92 Biscéré T, Ferrier-Pagès C, Grover R, Gilbert A, Rottier C, Wright A et al. Enhancement

93 Crossland C, Barnes D. The role of metabolic nitrogen in coral calcification. Mar Biol.

94 Jorgensen NOG. Uptake of urea by estuarine bacteria. Aquat Microb Ecol. 2006; 42: 227-242.

95 Pernice M, Raina J-B, Rädecker N, Cárdenas A, Pogoreutz C, Voolstra CR. Down to the bone: the role of overlooked endolithic microbiomes in reef coral health. ISME J. 2019: 1-10. an important role in the chemotactic motility of Helicobacter pylori in a viscous An information aesthetic for comparative genomics. Genome Res. 2009; 19: 1639-1645. 


\section{$915 \quad$ Figure Legends}

916 Figure 1. The phylogeny and population differentiation of the Ruegeria population. (A) The

917 rooted maximum-likelihood phylogenomic tree of the 20 strains isolated from the coral species

918 Platygyra acuta (Accession numbers were showed in Table S6). This tree is rooted with the

919 mid-point rooting method. Solid circles at the nodes indicate that the support value (IQ-TREE

920 v1.6.5 ultrafast bootstraps) of the branch is $100 \%$. The LCA of clade-M, the LCA of the five

921 mucus strains within the clade-M, and the LCA of clade-S are shown as blue triangle, the red star,

922 and the pink triangle, respectively. Strains isolated from different coral compartments are

923 highlighted with different colors. The clade-M and clade-S are also labeled. (B) The heatmap of

924 the whole-genome average nucleotide identity (ANI) and heatmap of the pairwise identity of $16 \mathrm{~S}$

925 rRNA genes of the 20 strains. (C) fineSTRUCTURE coancestry matrix of the 12 isolates from

926 clade-M and clade-S, with warmer colors representing more ancestry shared between strains

927 under comparison. Strains assigned to the same fineSTRUCTURE population are connected with

928 a vertical bar on the left of the matrix, and the dendrogram shows the clustering of the

929 fineSTRUCTURE populations. (D) Heatmap of accessory gene content similarity measured by

930 Jaccard distance, with warmer colors representing the higher similarity between strains. The left

931 dendrogram was generated based on the complete-linkage clustering method.

932

933 Figure 2. The genomic differentiation of the Ruegeria population. (A) The phylogeny and

934 pangenome of the Ruegeria population generated using Circos v0.64 [103]. The gene families

935 are arranged in order of the closed genome of strain HKCCD4315. The circular tracks depict the

936 genomes which are arranged in order of the phylogeny and shown in grey. From the inner to the

937 outer circle: (1)-(6) six genomes of clade-M. The genes gained at the LCA of clade-M are shown 
938 in cyan, and those lost at the LCA of clade-S are shown in blue. (7)-(12) Six genomes of clade-S.

939 The genes gained at the LCA of clade-S are shown in magenta, and those lost at the LCA of

940 clade-M are shown in orange. (13)-(20) Eight genomes of outgroup. The 536 clade-M specific

941 genes and the 365 clade-S specific genes are shown in yellow in all tracks. The genomic islands

942 (GIs) are showed in dark grey in all tracks. Other genes are shown in light grey in all tracks. (21)

943 The genomic region of the chromosome and three plasmids are shown in grey, and the core

944 genes showing unusually large synonymous substitution rate $\left(d_{s}\right)$ are shown in green.

946 Figure 3. The catabolic pathways of methylamine-related coral osmolytes in the Ruegeria

947 population. The compounds in grey blocks are osmolytes in coral. The solid arrows represent the

948 genes present in the Ruegeria population. The arrows in dashed lines represent the genes missing

949 from the Ruegeria population. The cyan arrows represent the clade-M specific genes. The green

950 arrows denote the outlier core genes showing unusually large between-clade $d_{S}$ values. Divergent

951 allelic replacement at the LCA of clade-M and clade-S are marked with solid green and red

952 circles, respectively. The open circle in green represents multiple allelic replacements within

953 clade-M. The black circles indicate that the allelic replacement history cannot be resolved based

954 on the available data. The black arrows show the core genes shared by all clade-M and clade-S

955 members. The red arrows indicate the reactions related to nitrogen assimilation. The yellow

956 arrows indicate the reactions related to carbon assimilation. There are no clade-S specific genes

957 found in these pathways. Only the dominant substrates of the promiscuous transporters are

958 shown in the figure. The conversion from GBT to TMA was previously revealed by a

959 metabolomics study on intestinal microbiota [67], but the underlying gene for this reaction has

960 not been known. 
962 Figure 4. Growth experiments of three clade-M strains and three clade-S strains. (A) The

963 minimal medium is supplemented with different substrates. (1) Control experiments. In the

964 negative control, bacteria are cultured in the minimal medium without $\mathrm{C}$ and $\mathrm{N}$ source (open

965 circles). In the positive control, bacteria are cultured in the rich medium, in which the peptone,

966 glucose and yeast extracts are added as mixed C and N sources (open triangles). Growth

967 experiments of the six strains on $5 \mathrm{mM}$ of choline (2), GBT (3), DMG (4), sarcosine (5), TMA

968 (6), TMAO (7), creatine (8), L-proline (9), taurine (10) and urea (11), each used as a sole C and

969 N source (solid triangles). Three replicates are performed for each condition and error bars

970 denote standard deviation. (B) Different substrates are used as either a sole C source or a sole $\mathrm{N}$

971 source. (1) Control experiments. In the negative control, bacteria are cultured in the minimal

972 medium without $\mathrm{C}$ and $\mathrm{N}$ source added (open circles). In the positive control, bacteria are

973 cultured using pyruvate as the $\mathrm{C}$ source and ammonium as the $\mathrm{N}$ source, respectively (open

974 triangles). Growth experiments of the six strains on $5 \mathrm{mM}$ of DMSP (2), choline (3), GBT (5),

975 TMA (7), TMAO (9) and urea (11) as the sole C source and each with ammonium (10 mM) as

976 the $\mathrm{N}$ source (solid triangles). Growth experiments of six strains on $5 \mathrm{mM}$ of choline (4), glycine

977 betaine (6), TMA (8), TMAO (10) and urea (12) as the sole $\mathrm{N}$ source and each with pyruvate (5

$978 \mathrm{mM}$ ) as the $\mathrm{C}$ source (solid triangles). Three replicates are performed for each condition and

979 error bars denote the standard deviation.

981 Figure 5. The phylogenetic distribution and pseudogene characterization of the flagellar gene

982 cluster flal across the expanded Ruegeria population composed of isolates from both coral and 
983 non-coral marine habitats, along with the motility assays of select isolates. (A) The gene

984 arrangement of the flal clusters in members of exp-clade-M and exp-clade-S. The pink arrows

985 represent the clade-S specific genes, and the grey arrows denote core genes shared by

986 exp-clade-M and exp-clade-S, respectively. The pseudogenes are marked with red crosses on the

987 arrows. (B) The motility assays of three clade-M strains and three clade-S strains. Swimming and

988 swarming motilities are tested on $0.3 \%(\mathrm{w} / \mathrm{v})$ soft agar and $0.6 \%(\mathrm{w} / \mathrm{v})$ agar for eight days,

989 respectively; twitching motility is tested on $1.0 \%(\mathrm{w} / \mathrm{v})$ agar in the humidified box for 10 days.

990 (C) An expanded genome phylogeny of the Ruegeria population based on 44 strains including

991 those collected from various ecological niches. The exp-clade-M, exp-clade-S and exp-outgroup

992 expand from the clade-M, clade-S and outgroup in Fig. 1A. Solid circles at the nodes indicate

993 that the ultrafast bootstrap support value of the branch is $100 \%$. The scale bar indicates the

994 number of substitutions per site. The root of the tree is determined using a larger phylogenomic

995 tree (Fig. S12) composed of 454 Ruegeria and related strains [50]. The strains containing the

996 complete flal cluster are marked with black boxes. The strains with partial flal clusters are

997 marked with white boxes, and different types of partial flal clusters in exp-clade-M and

998 exp-clade-S are distinguished by the numbers in the white boxes.

999

1000 Figure 6. Compartmentalization of two subpopulations of the Rhodobacteraceae population

1001 each from a distinct coral individual. (A-B) Phylogenomic trees constructed by IQ-TREE v1.6.5

1002 using the core SNPs identified with kSNP3 for the population associated with the coral

1003 individual collected at Wong Wan Chau and the other associated with the coral individual at Ngo

1004 Mei Chau. Strains isolated from different coral compartments are highlighted with different 
1005 colours. Solid circles at the nodes indicate that the ultrafast bootstrap support value of the branch

1006 is $\geq 80 \%$. The scale bar indicates the number of SNPs per variable site. (C-D) The frequency

1007 distribution of the number of migrations required to produce each of the 100,000 permuted trees

1008 in the two subpopulations. The blue arrow denotes the inferred number of migrations based on

1009 the real data. 


\section{Figure 1}

A

B

Seawater:

Mucus:

Tissues:

Skeleton:

Tree scale:

$0.01 \longmapsto$

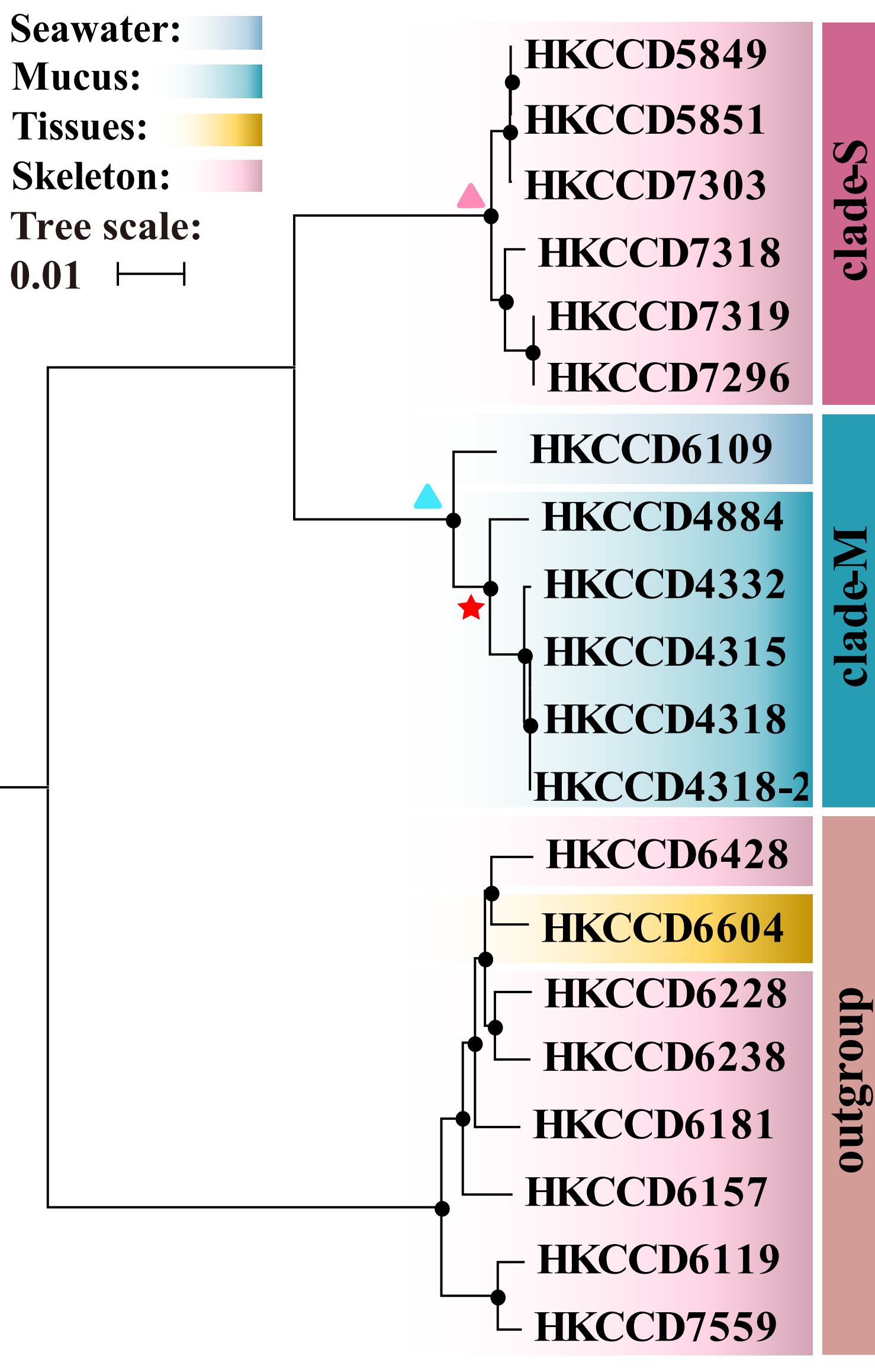

HKCCD5849

HKCCD7319

HKCCD7296

$\mathrm{C}$

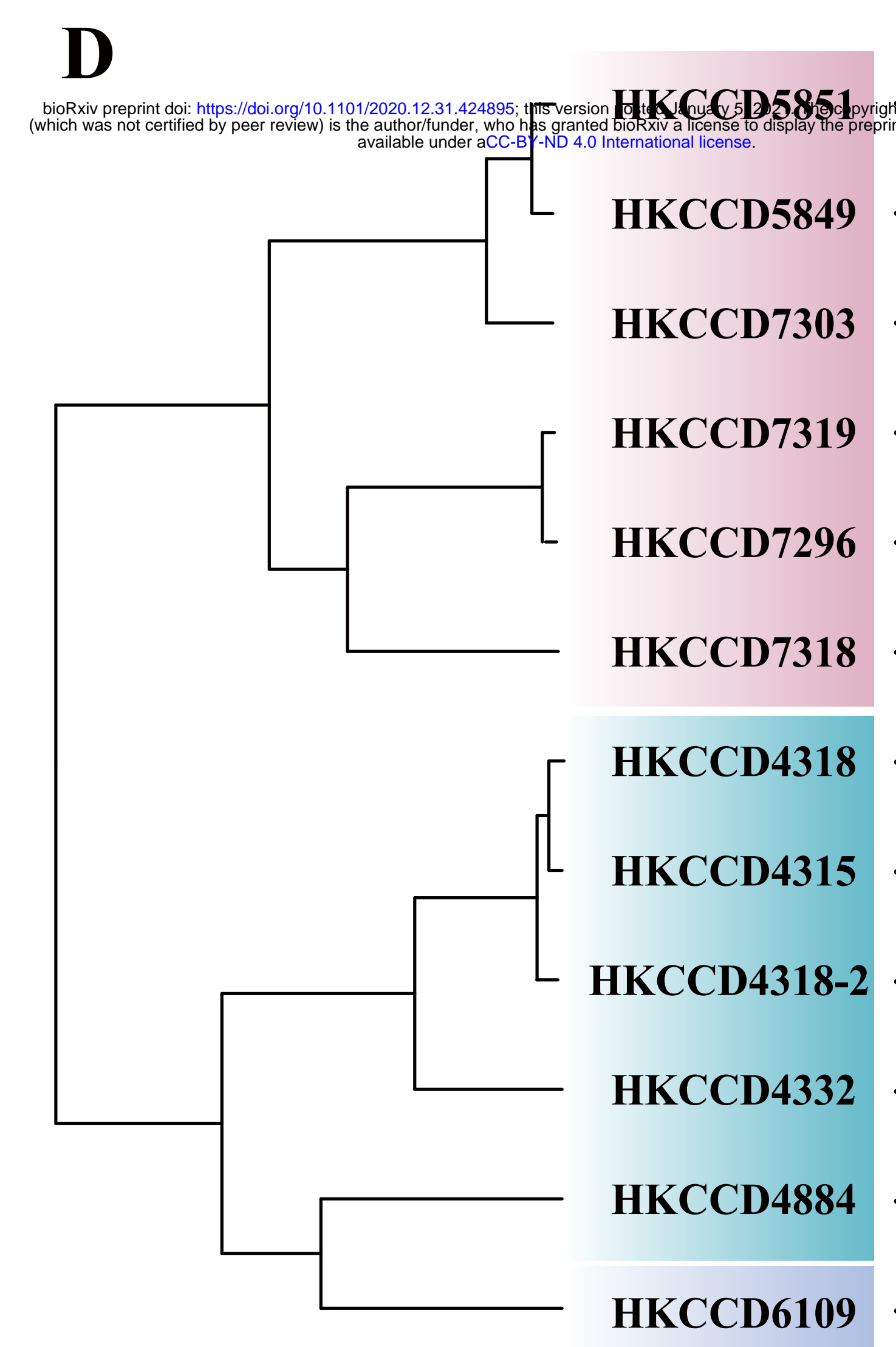

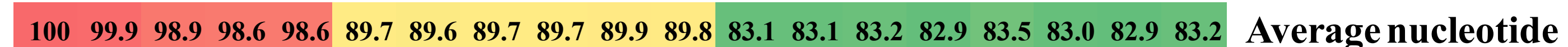

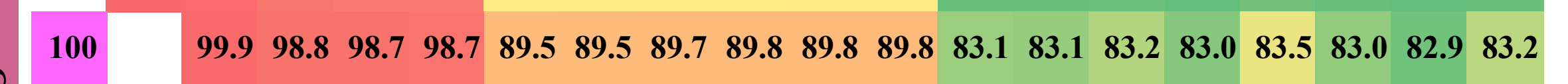

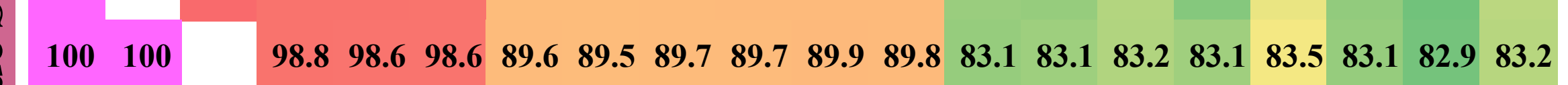

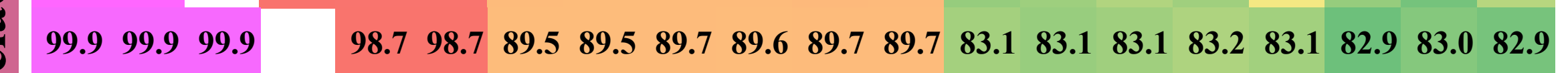

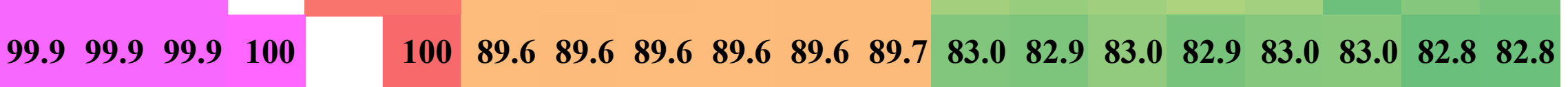
\begin{tabular}{llll|lllllllllllllll}
99.9 & 99.9 & 99.9 & 100 & 100 & 89.7 & 89.6 & 89.6 & 89.6 & 89.7 & 89.8 & 83.0 & 83.0 & 83.1 & 82.9 & 83.0 & 83.0 & 82.8 & 82.9
\end{tabular}

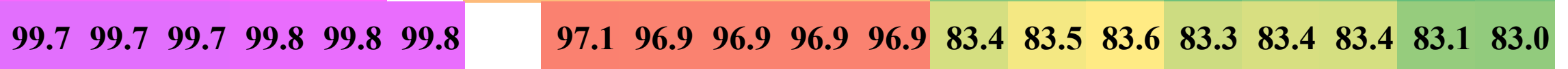

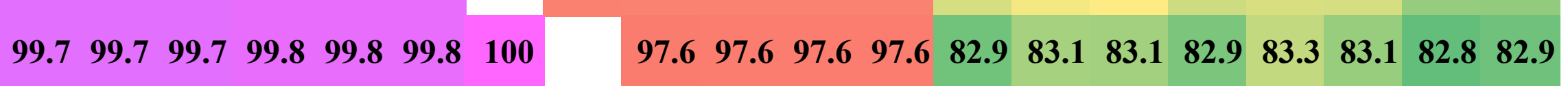

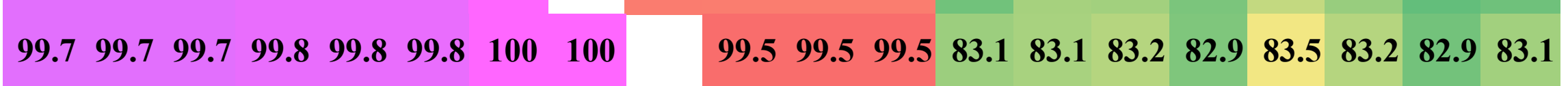

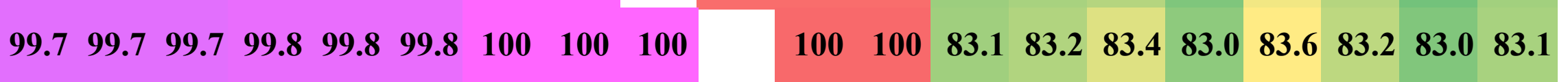

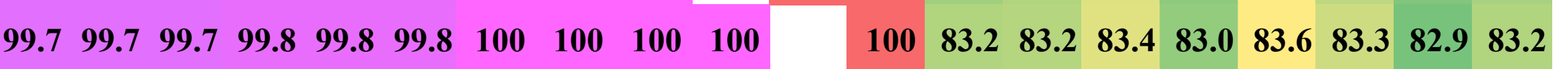

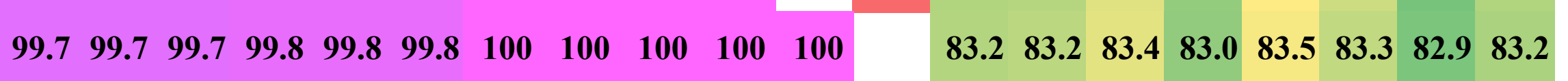

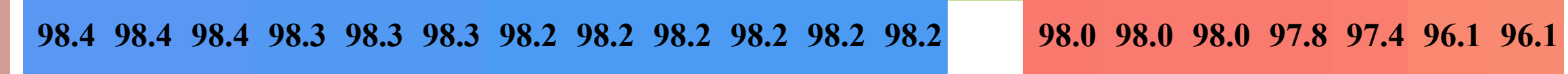

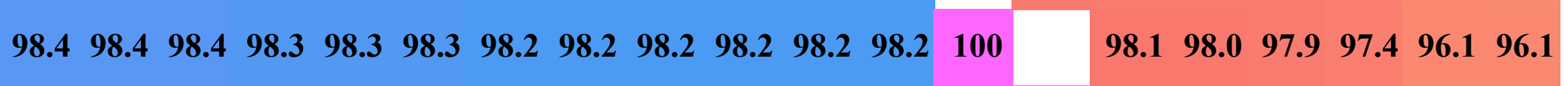

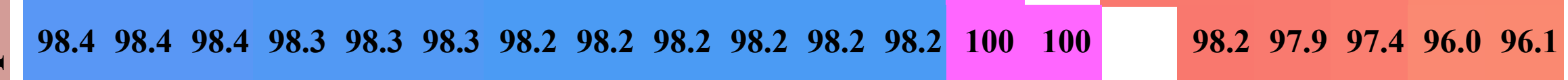

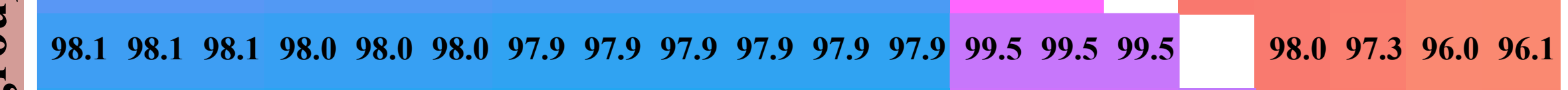
97.9 97.9 97.9 97.9 97.9 97.9 97.8 97.8 97.8 97.8 97.8 97.8 99.5 99.5 99.5 99.4 $\quad$ 97.4 96.0 96.2 97.6 97.6 97.6 97.5 97.5 97.5 97.5 97.5 97.5 97.5 97.5 97.5 99.2 99.2 99.2 98.8 98.7 $\quad 96.196 .1$

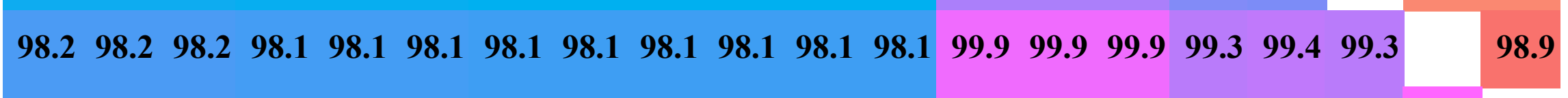
98.2 98.2 98.298.1 98.1 98.198.198.1 98.1 98.198.1 98.1 99.9 99.9 99.9 99.3 99.4 99.3 100

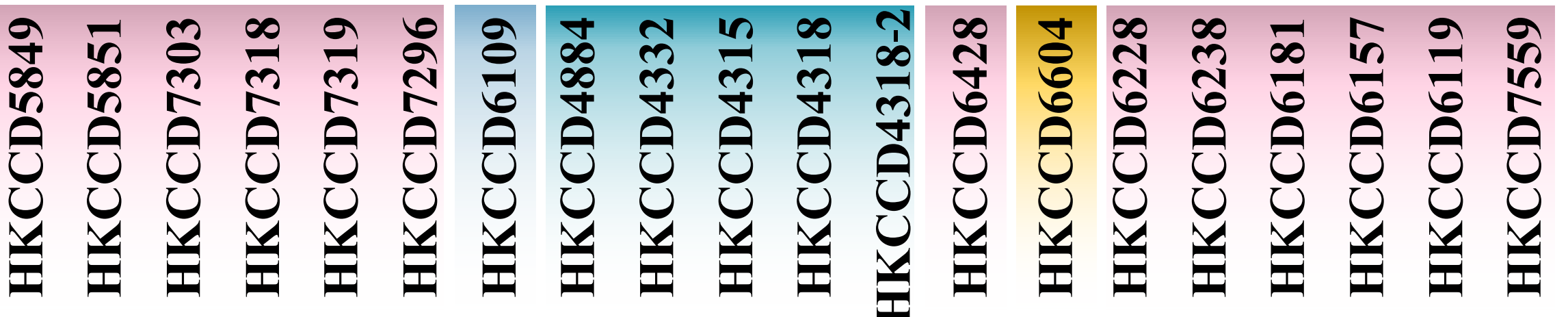
rRNA genes: $\left.\begin{array}{c}100 \\ 99.5 \\ 99.0 \\ 98.5 \\ 98.0 \\ 97.5\end{array}\right]$

Coancestry:

$\left.\begin{array}{l}4430 \\ 3930 \\ 3440 \\ 2950- \\ 2460 \\ 1970 \\ 1480 \\ 986 \\ 495- \\ 3.3\end{array}\right]$

Similarity of accessory gene content:
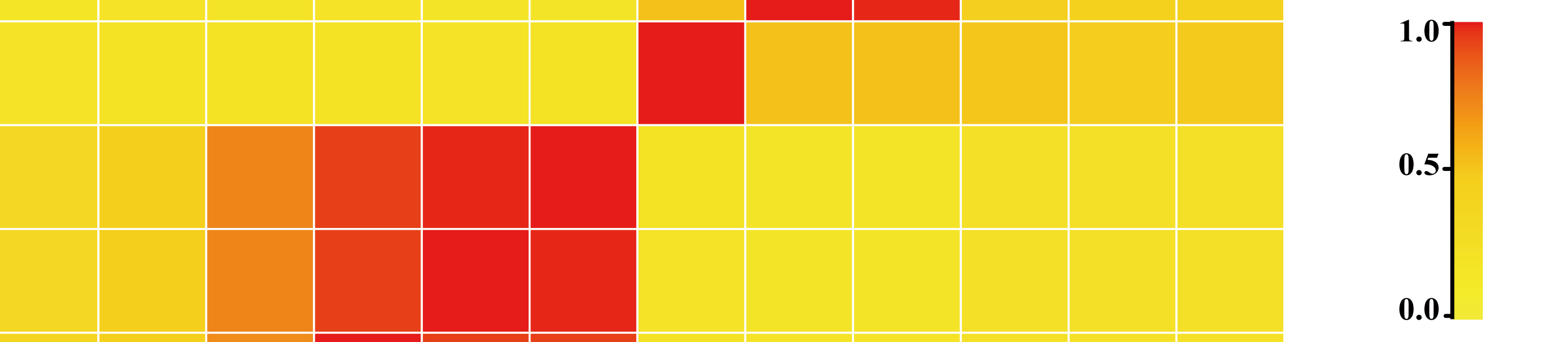


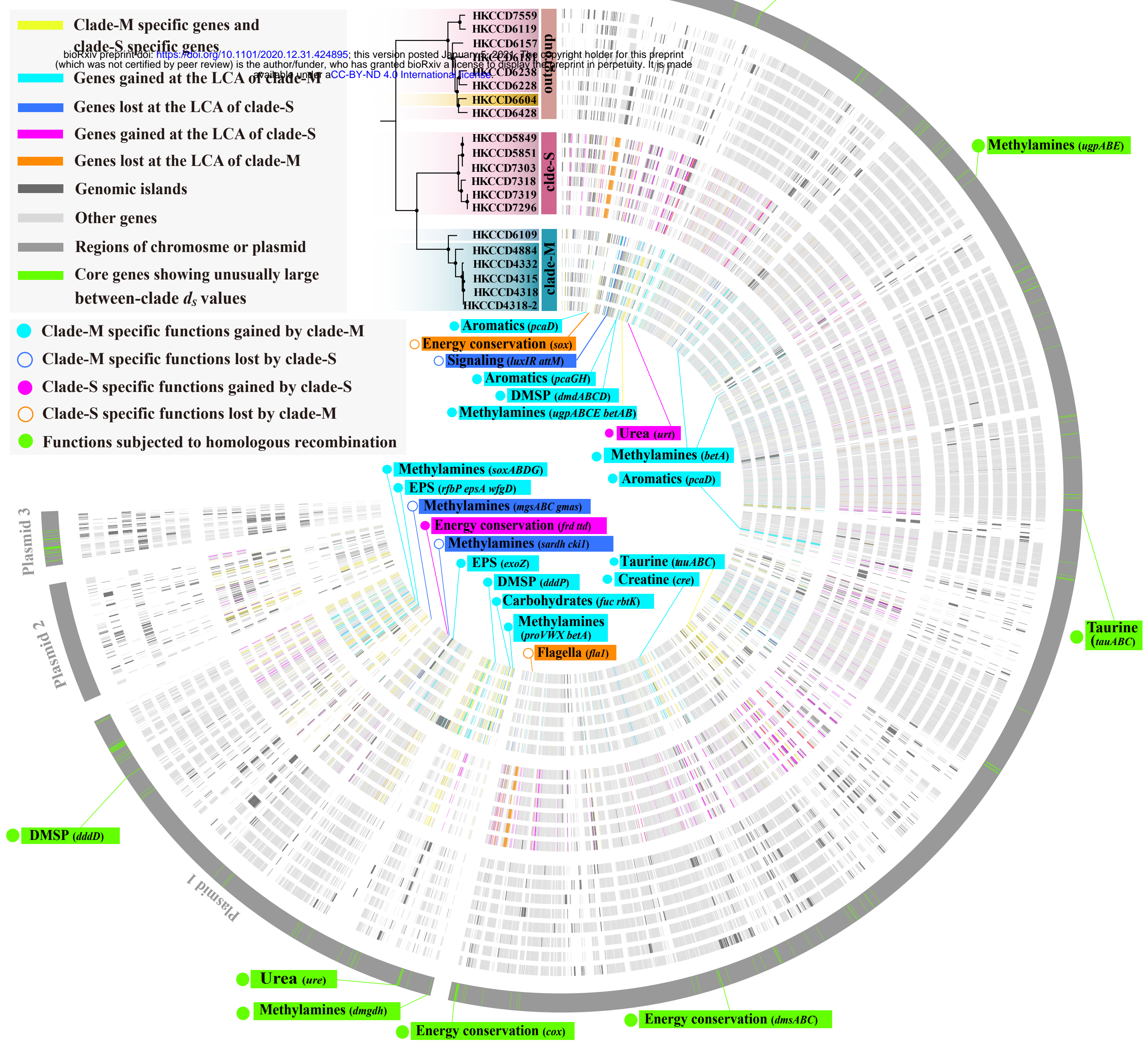




\section{Figure 4}
A — HKCCD4315
HKCCD5849
- HKCCD7318
$-H K C D$
$\triangle$ Substrates used as sole $\mathrm{C}$ and $\mathrm{N}$ sources
- HKCCD6109
- HKCCD7319
$\Delta$ Rich medium

(1) Controls
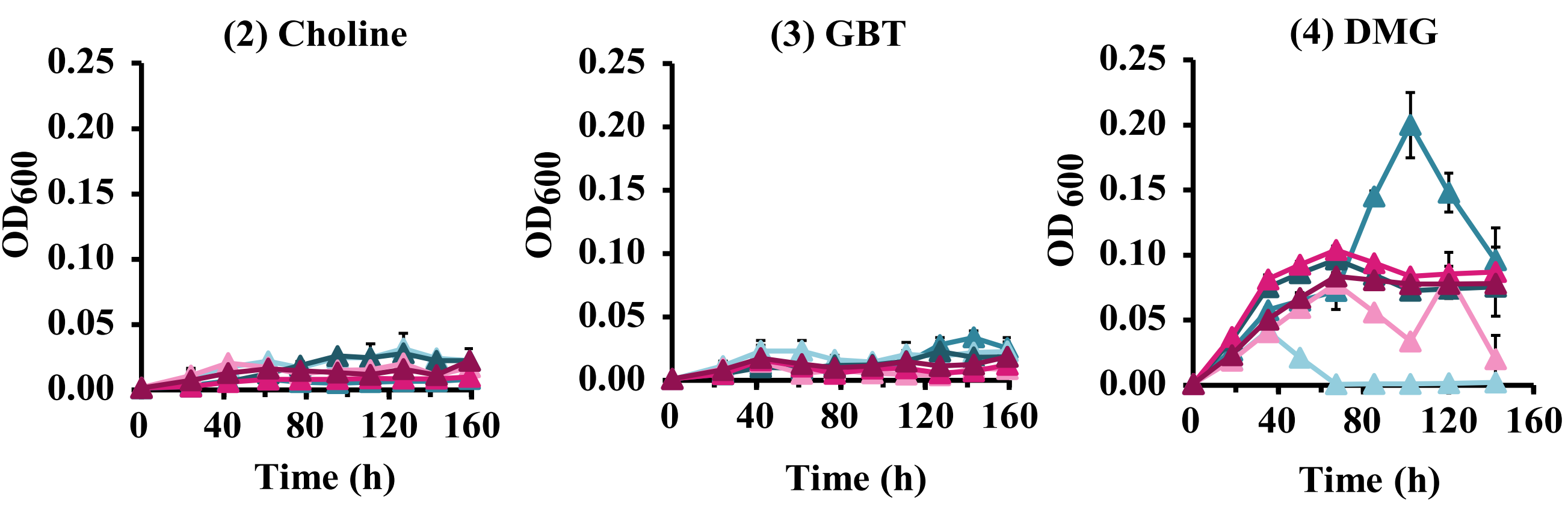

(5) Sarcosine

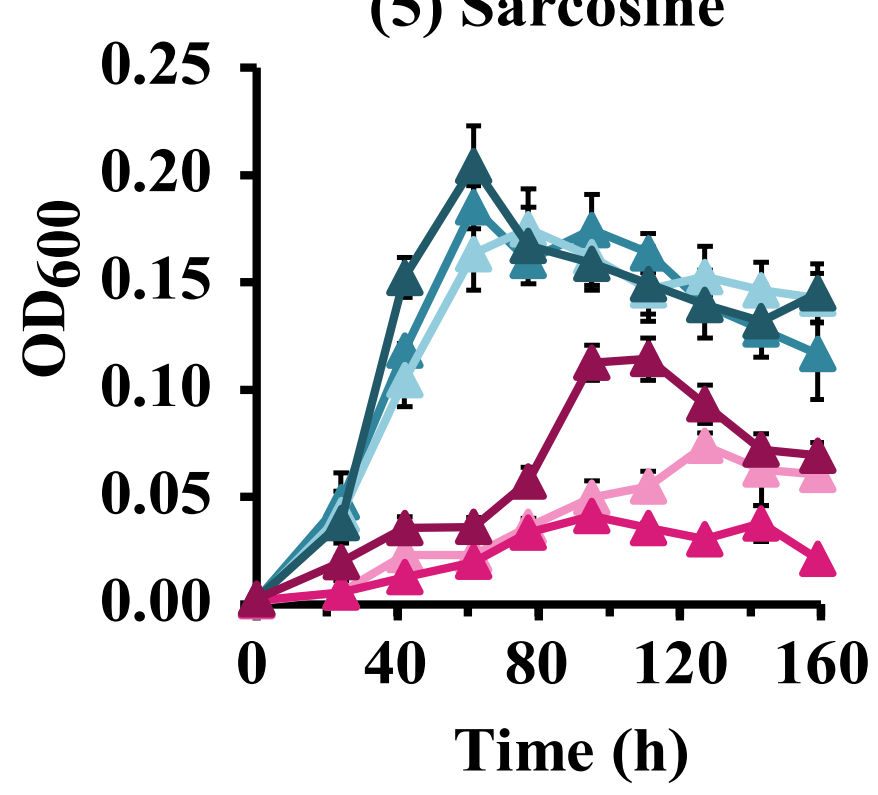

(6) TMA
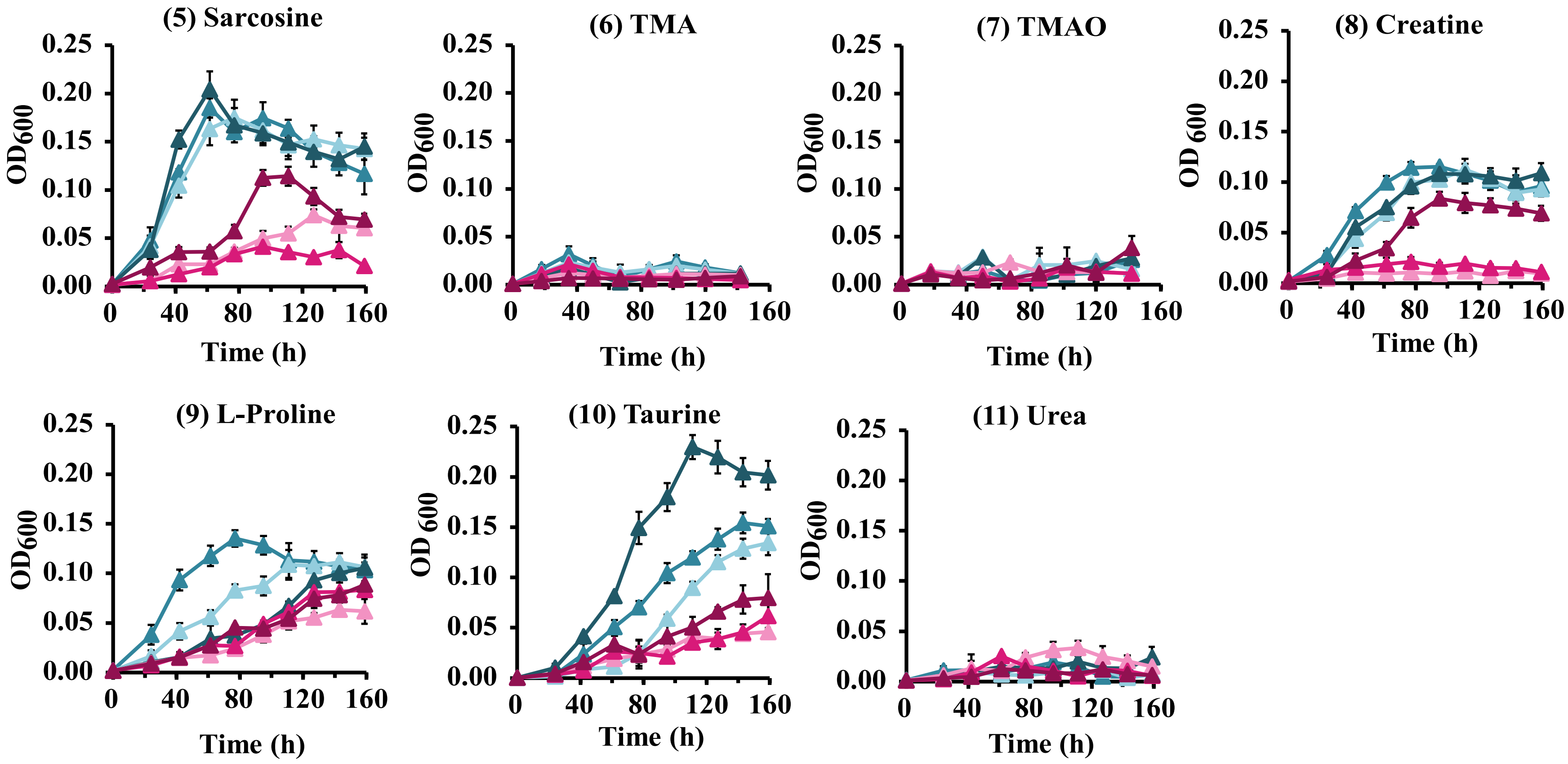

B $\triangle$ Pyruvate used as sole $\mathrm{C}$ source or ammonium used as sole $\mathrm{N}$ source

$\Delta$ Rich medium

O No C and N sources

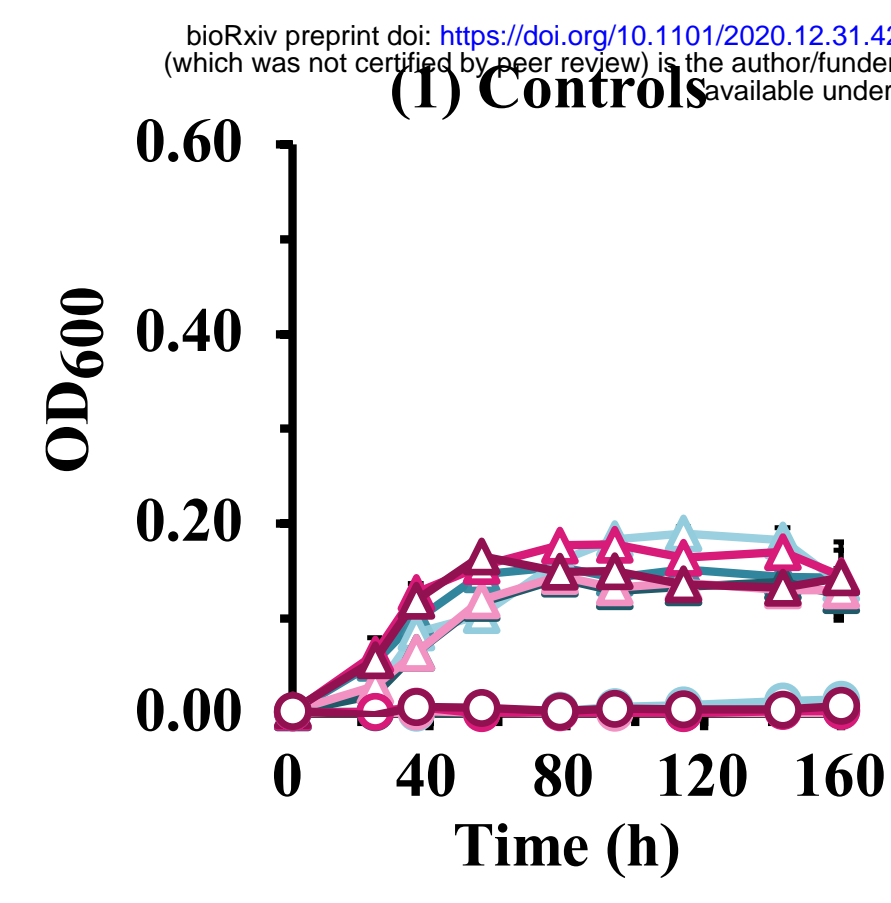

(5) GBT as sole

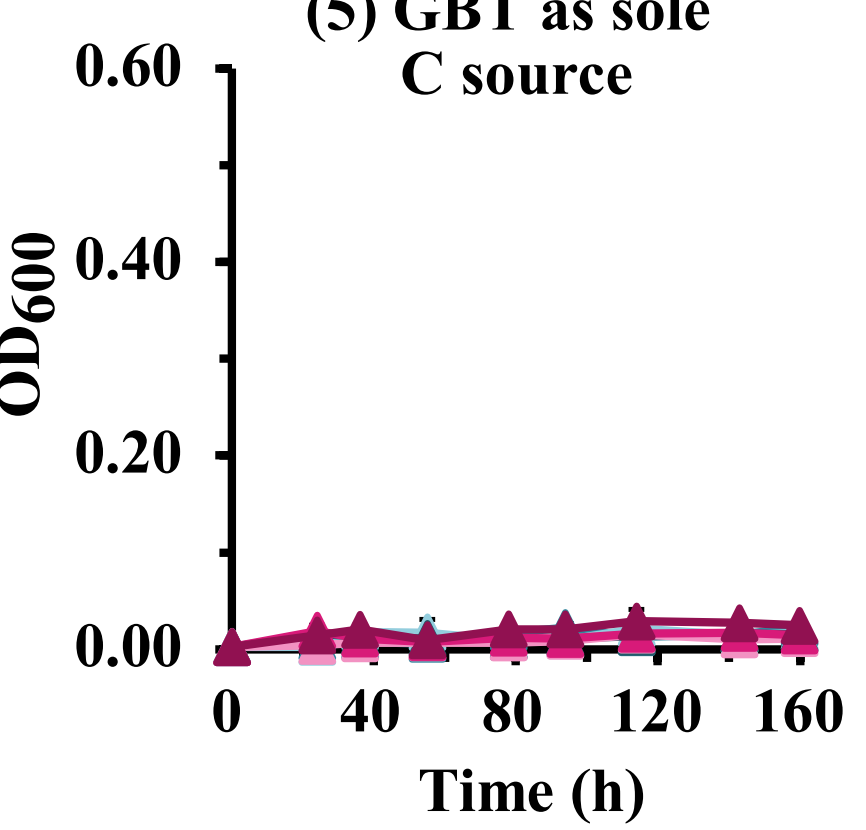

(9) TMAO as sole

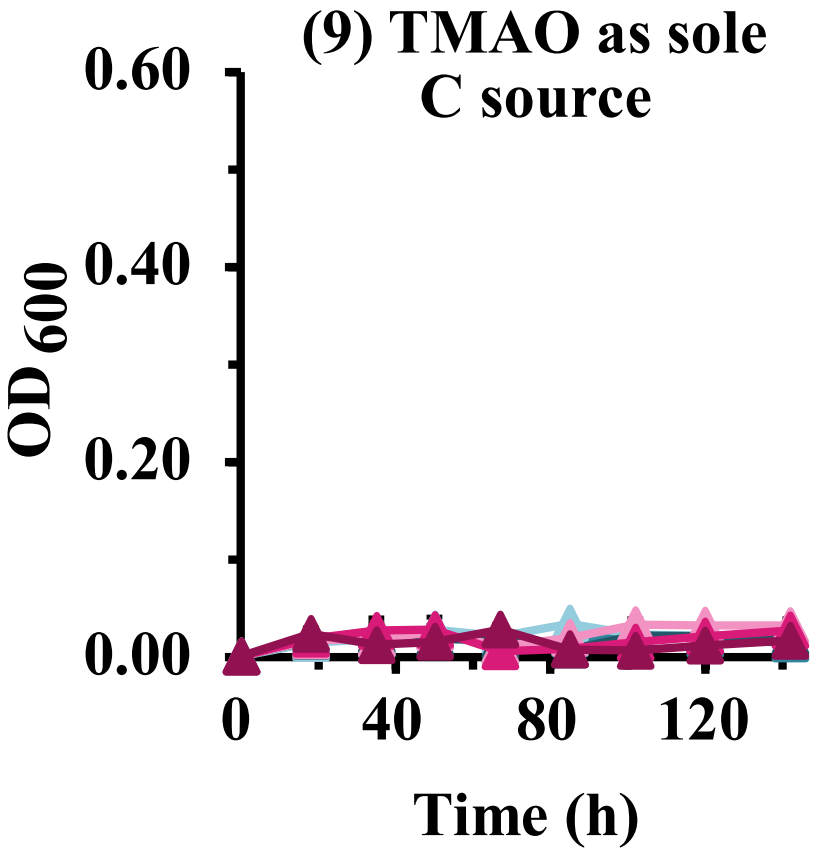

(3) Choline as sole
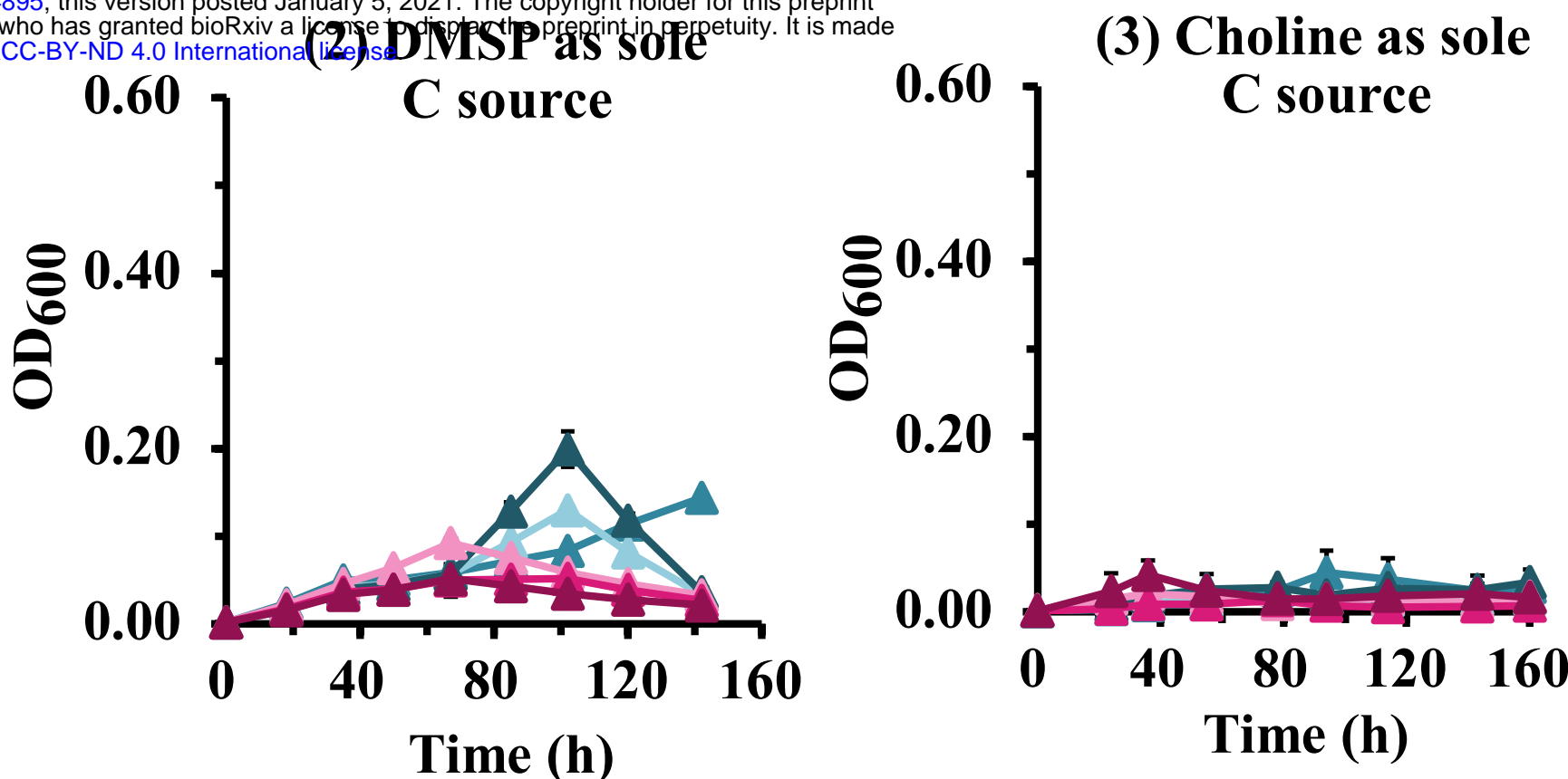

(6) GBT as sole
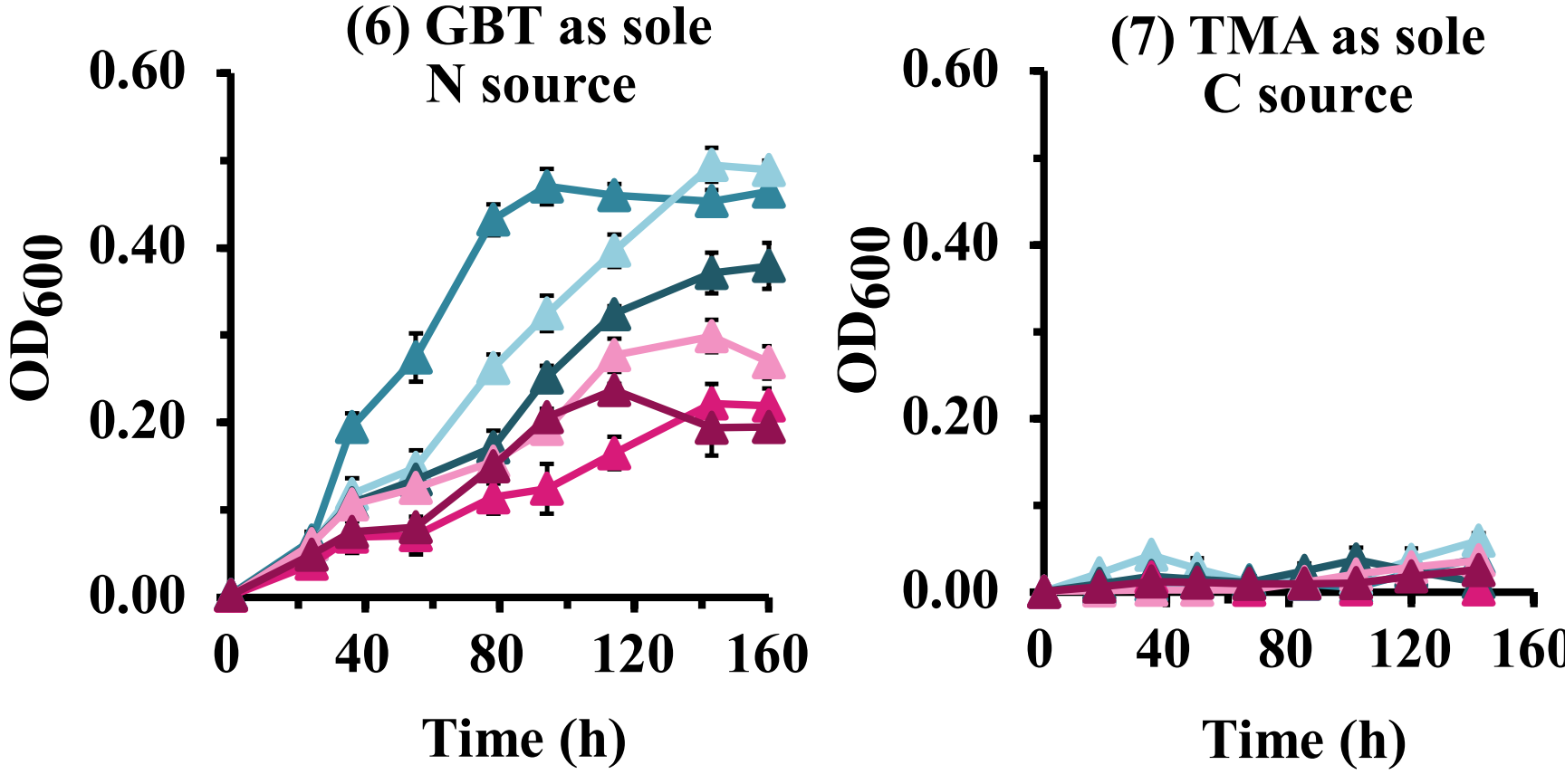

(10) TMAO as sole

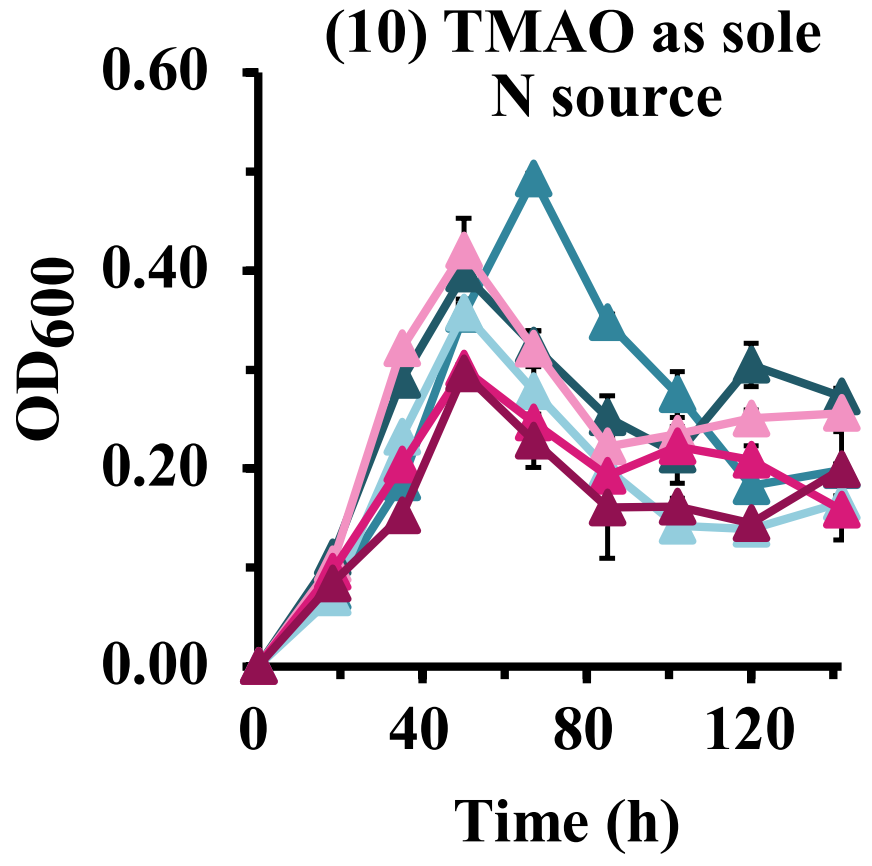

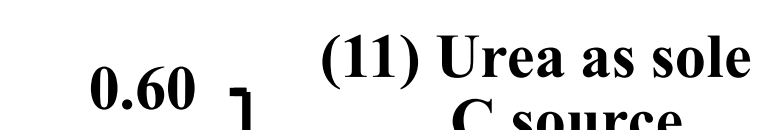

Urea as so
C source

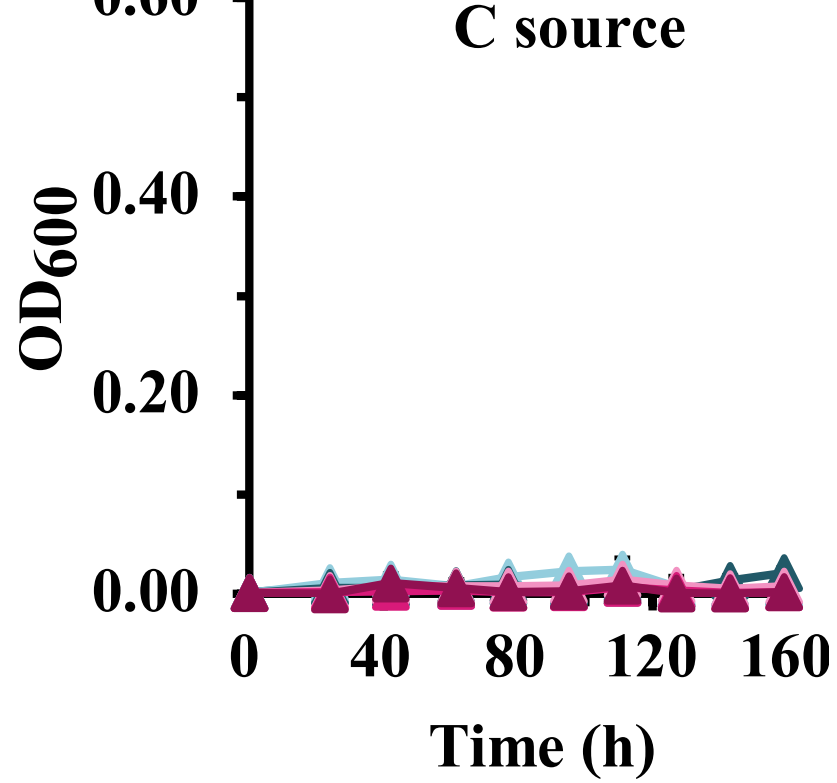

(4) Choline as sole

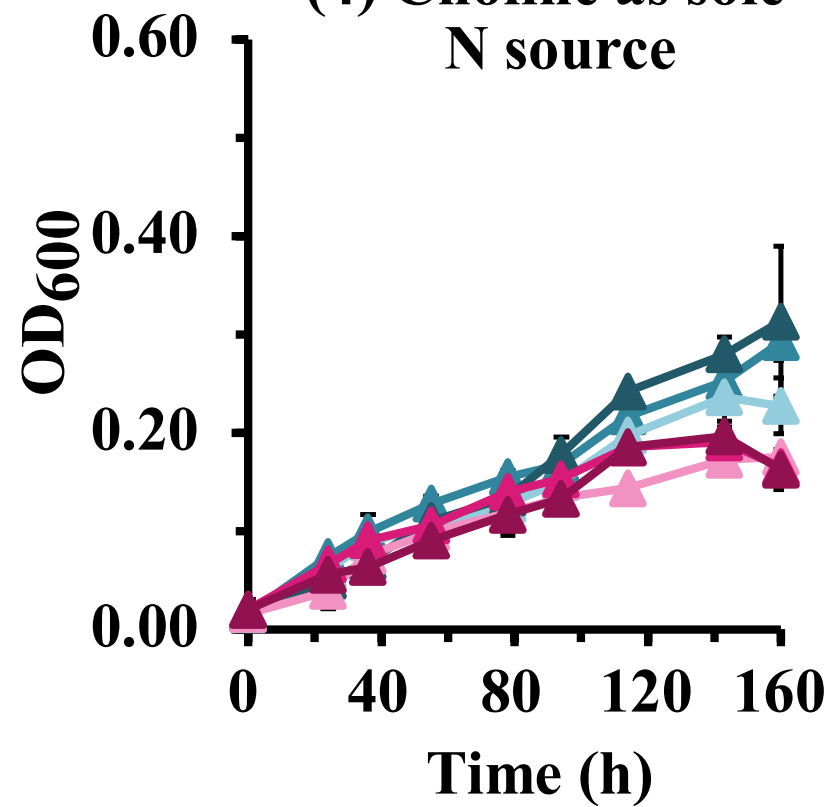

(8) TMA as sole N source
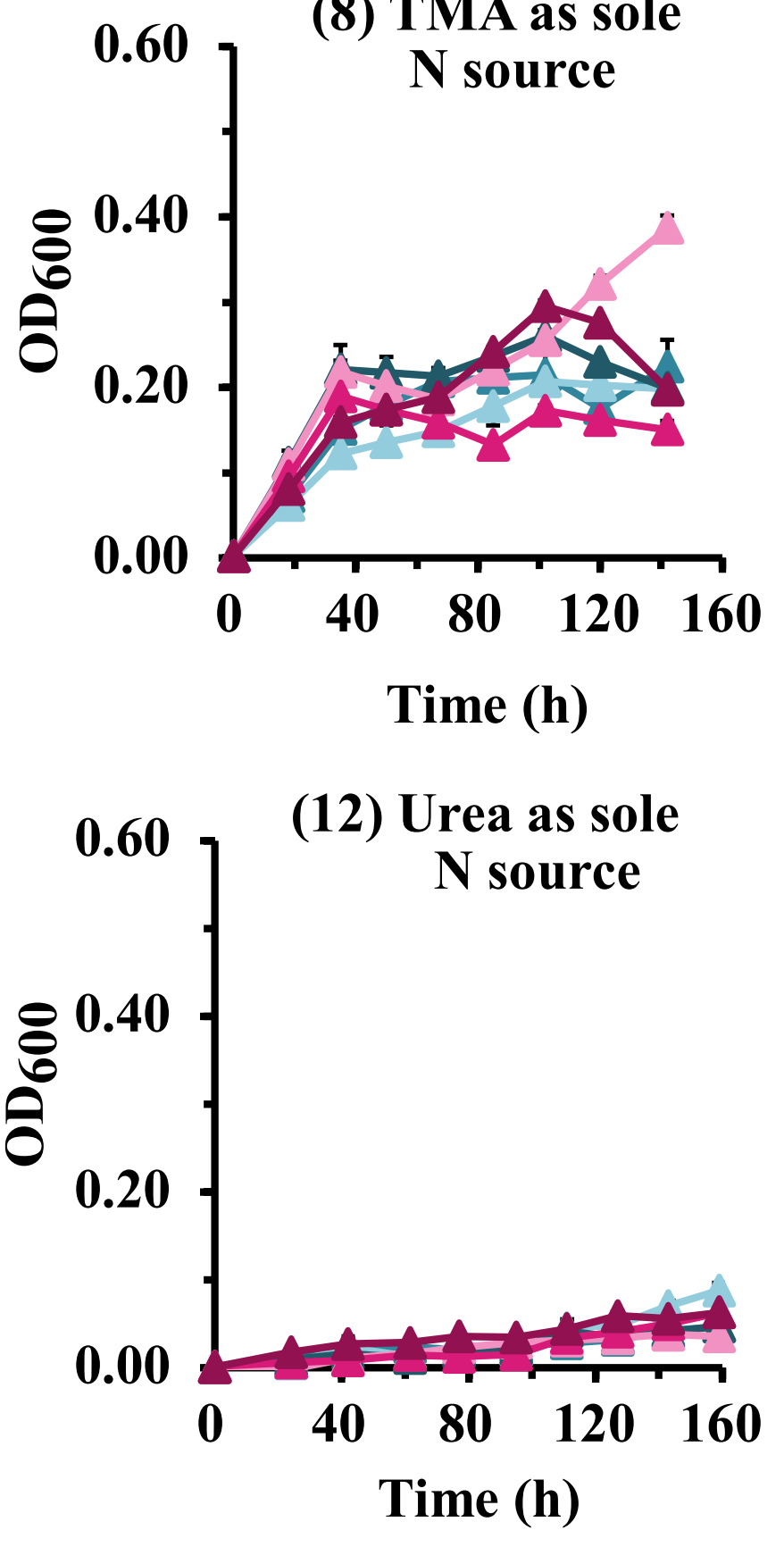


\section{A. Wong Wan Chau}

\section{B. Ngo Mei Chau}

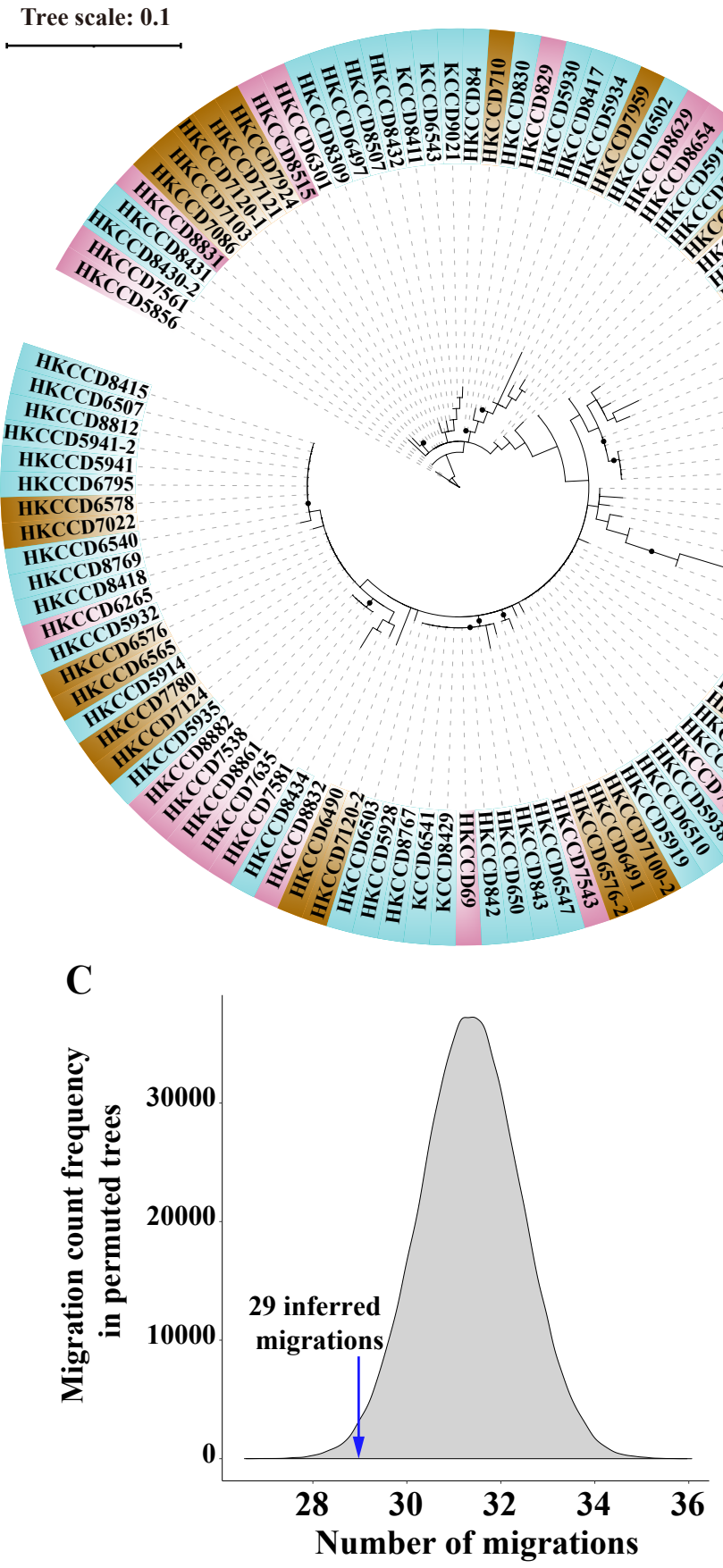

Mucus

Tissues

Skeleton

Tree scale: 0.1

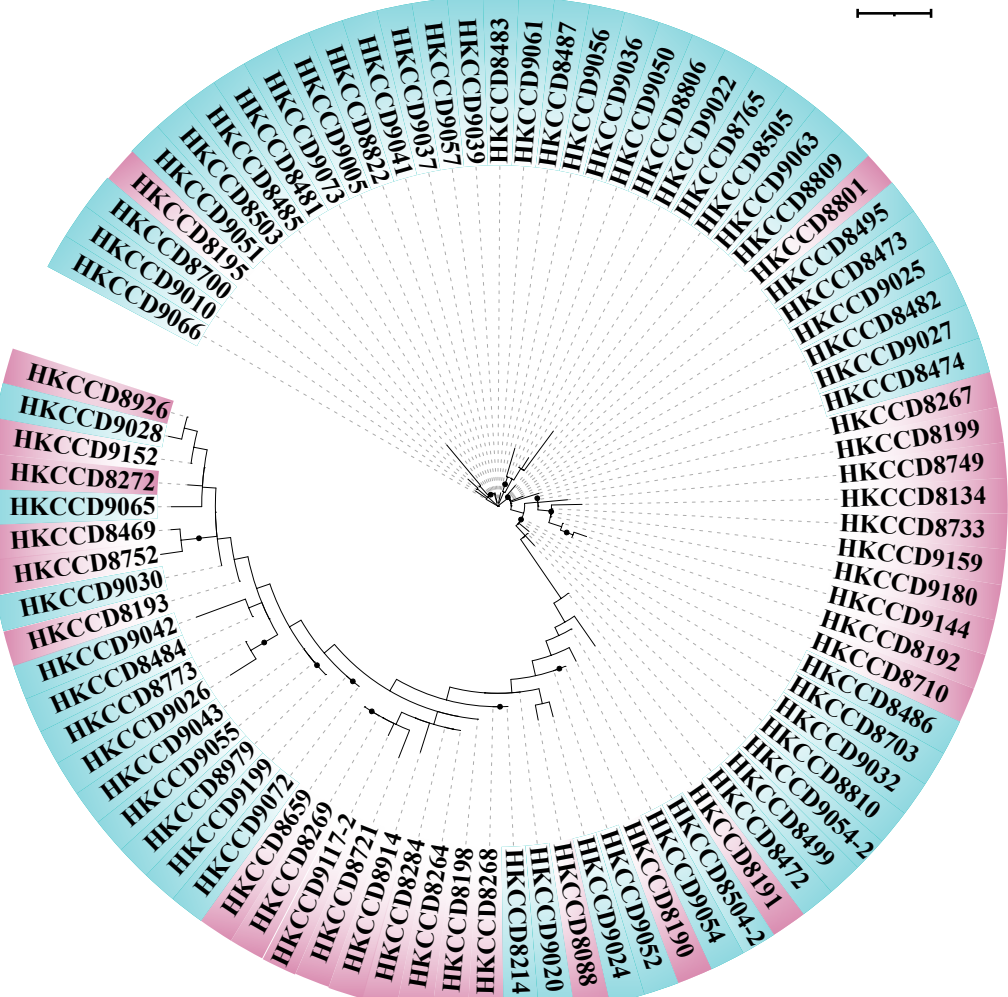

D

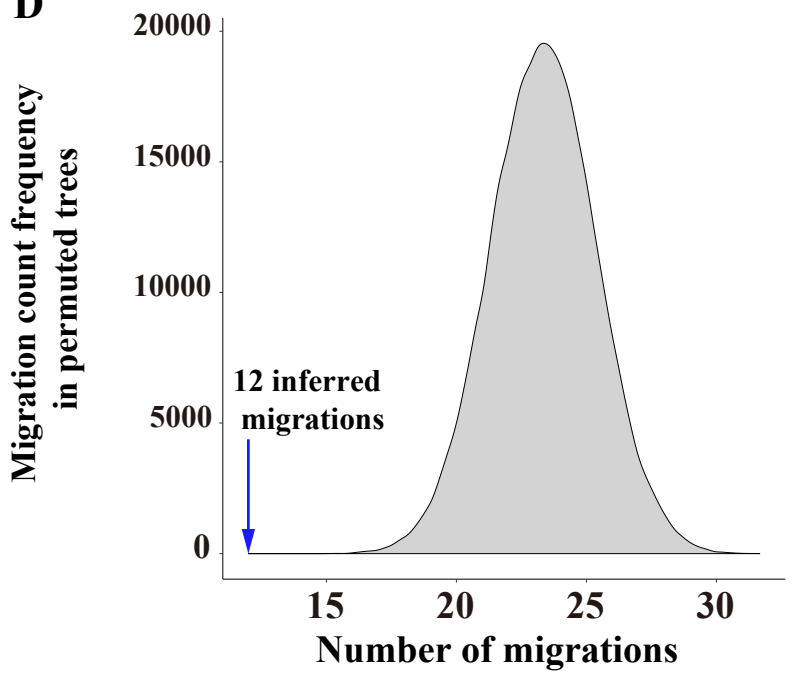


bioRxiv preprint doi: https://doi.org/10.1101/2020.12.31.424895; this version posted January 5, 2021. The copyright holder for this preprint (which was not certified by peer review) is the author/funder, who has granted bioRxiv a license to display the preprint in perpetuity. It is made available under aCC-BY-ND 4.0 International license.

Table. 1 Genetic variation of the Ruegeria population and the Rhodobacteraceae population.

\begin{tabular}{clccccc}
\hline \multirow{2}{*}{ Population } & Clade & \#strains & $\begin{array}{c}\text { 16S rRNA gene identity } \\
\text { (mean } \pm \text { sd) }\end{array}$ & $\begin{array}{c}\text { ANI } \\
\text { (mean } \pm \text { sd) }\end{array}$ & \#SNP/Mbp & \#SNP \\
\hline Ruegeria & clade-M & 6 & $100.00 \pm 0.00$ & $98.24 \pm 1.32$ & 36,976 & 175,214 \\
& clade-S & 6 & $99.96 \pm 0.04$ & $99.05 \pm 0.57$ & 16,606 & 75,989 \\
& outgroup & 8 & $99.52 \pm 0.36$ & $97.08 \pm 0.94$ & 131,482 & 564,323 \\
& clade-M versus clade-S & 12 & $99.76 \pm 0.03$ & $89.67 \pm 0.1$ & 106,078 & 502,661 \\
& clade-M versus outgroup & 14 & $98.00 \pm 0.25$ & $83.04 \pm 0.16$ & 116,843 & 553,672 \\
& clade-S versus outgroup & 14 & $98.10 \pm 0.25$ & $83.18 \pm 0.21$ & 117,499 & 537,689 \\
Rhodobacteraceae & - & 214 & $100.00 \pm 0.00$ & $99.99 \pm 0.64$ & 10 & 43 \\
\hline
\end{tabular}

